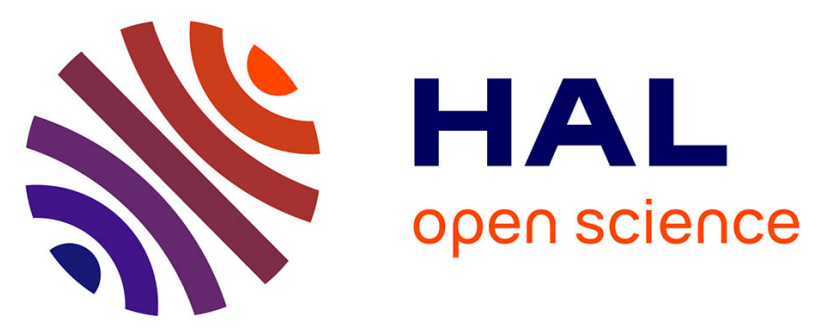

\title{
Géoarchéologie et taphonomie des vestiges archéologiques: impacts des processus naturels sur les assemblages et méthodes d'analyse
}

Pascal Bertran, Jean-Guillaume Bordes, Dominique Todisco, Luc Vallin

\section{- To cite this version:}

Pascal Bertran, Jean-Guillaume Bordes, Dominique Todisco, Luc Vallin. Géoarchéologie et taphonomie des vestiges archéologiques: impacts des processus naturels sur les assemblages et méthodes d'analyse. sous la direction de Jean-Philip Brugal. TaphonomieS. Ouvrage du Groupement de recherches "Taphonomie, Environnement et Archéologie", CNRS-INEE, Editions des Archives Contemporaines, pp.123-156, 2017, 9782813002419. hal-01696670

\section{HAL Id: hal-01696670 https://hal-inrap.archives-ouvertes.fr/hal-01696670}

Submitted on 6 Feb 2018

HAL is a multi-disciplinary open access archive for the deposit and dissemination of scientific research documents, whether they are published or not. The documents may come from teaching and research institutions in France or abroad, or from public or private research centers.
L'archive ouverte pluridisciplinaire $\mathbf{H A L}$, est destinée au dépôt et à la diffusion de documents scientifiques de niveau recherche, publiés ou non, émanant des établissements d'enseignement et de recherche français ou étrangers, des laboratoires publics ou privés. 


\title{
Géoarchéologie et taphonomie des vestiges archéologiques : impacts des processus naturels sur les assemblages et méthodes d'analyse
}

\author{
Pascal Bertran ${ }^{1,2}$, Jean-Guillaume Bordes ${ }^{2}$, \\ Dominique Todisco ${ }^{3}$, Luc Vallin ${ }^{4}$ \\ 1 INRAP \\ ${ }^{2}$ UMR 5199, PACEA, CNRS - Université de Bordeaux \\ 3 UMR 6266, IDEES, Département de géographie, Université de Rouen \\ ${ }^{4}$ Service régional de l'archéologie Nord-Pas-de-Calais
}

\section{Processus de formation des sites et taphonomie archéologique : principes généraux}

L'idée que tous les sites archéologiques, et notamment paléolithiques, ont subi à des degrés divers des transformations par les processus naturels après leur abandon par l'Homme s'est progressivement imposée surtout à partir des années 1980, à la suite d'un certain nombre de travaux de géoarchéologie et d'archéologie [1-10]. Ces transformations oblitèrent de façon plus ou moins profonde les caractéristiques initiales des niveaux d'occupation, tant en ce qui concerne la distribution spatiale des vestiges que l'intégrité et la composition des assemblages lithiques et osseux (figure 1). Elles font partie intégrante, au même titre que les processus anthropiques, des mécanismes de formation d'un site tel qu'il est découvert par l'archéologue. Le type d'information que ce dernier peut espérer extraire de l'étude archéologique des vestiges dépend donc très largement de l'intensité de ces transformations, intensité qui ne peut être considérée a priori comme négligeable mais nécessite d'être analysée dans le cadre d'une analyse critique ou « analyse taphonomique ». Tel que nous le définissons ici, le terme « taphonomie », qui désigne originellement « l'étude de la transition du matériel paléontologique de la biosphère à la lithosphère » [11], est utilisé par extension pour l'ensemble des vestiges indépendamment de leur nature (osseuse, mais aussi lithique, etc.), dans la mesure où, pour une grande part, les différents types de vestiges sont 


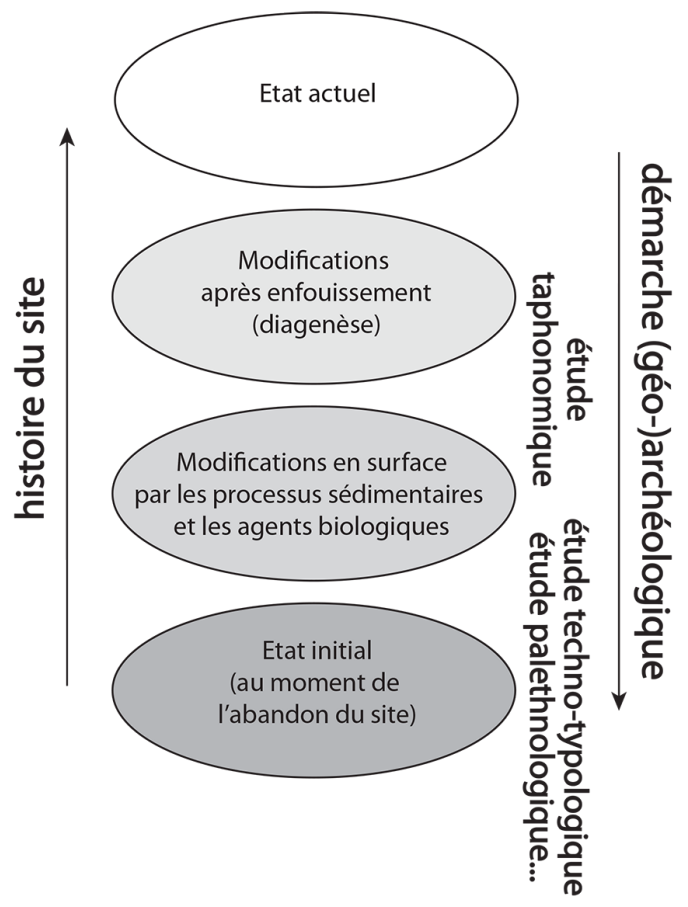

Figure 1 - Évolution d'un site et place de l'approche taphonomique.

affectés par des mécanismes d'enfouissement et de transformation identiques. Cette acception large revêt une dimension chronologique : elle s'attache à caractériser les processus qui ont affecté un ensemble de vestiges archéologiques postérieurement à leur abandon et analyse les conséquences de ces processus sur cet ensemble [12]. Une telle utilisation du terme taphonomie n'est cependant pas partagée par l'ensemble de la communauté, de nombreux paléontologues préférant se limiter à la définition princeps (voir notamment le débat entre [13] et [14]). L'expression de « taphonomie archéologique » a donc été utilisée pour distinguer cette démarche, devenue classique, de la discipline originelle.

Selon la conception exposée ci-dessus, la taphonomie concerne spécifiquement l'étude des processus de dégradation des vestiges et des structures archéologiques qui se produisent après le départ des hommes de leur lieu d'occupation. Elle contribue à isoler des ensembles de vestiges pour lesquels la cohérence chrono-culturelle aura été déterminée comme homogène et adaptée aux questions archéologiques posées. La démarche d'analyse taphonomique n'englobe pas l'ensemble des études géologiques et, plus largement, paléoenvironnementales qui sont habituellement réalisées sur les sites archéologiques, bien que celles-ci constituent une étape obligée dans l'analyse des processus de formation d'un site et qu'elles concourent donc largement à la compréhension des phénomènes de dégradation des ensembles archéologiques. Ce type d'études en contexte archéologique a donné lieu à une abondante littérature, notamment dans les 
revues consacrées au Quaternaire, qui ne sera que marginalement évoquée ici. Contrairement à l'analyse taphonomique proprement-dite, située à la charnière entre géologie et archéologie et qui nécessite donc une bonne connaissance des deux champs disciplinaires, les études paléoenvironnementales sont pratiquées par une communauté plus large de chercheurs issus de diverses branches des Sciences de la terre et du vivant ou des Sciences humaines (géographie).

L'analyse des facteurs de conservation des sites à l'échelle du paysage procède d'une démarche qui s'apparente par certains aspects à l'étude taphonomique intra-site. Ce domaine reste encore peu exploré en France. Ce point ne sera donc pas abordé dans le présent article.

La taphonomie archéologique intéresse bien évidemment tous les types de vestiges, auxquels d'ailleurs elle applique des outils méthodologiques communs. Ainsi, tous les outils que nous présenterons ici peuvent être appliqués à n'importe quel registre de vestiges. Nous nous appuierons cependant principalement sur les vestiges lithiques, afin de compléter les informations fournies pour la faune par les autres contributions à cet ouvrage. Suivant cette même logique, nous nous concentrerons sur les seuls vestiges lithiques lorsque nous parlerons de l'altération des matériaux. Les processus d'altération sont en effet largement dépendants de la nature des objets.

Deux grands types de transformations des niveaux archéologiques sont habituellement distingués : (1) les processus sédimentaires, qui affectent le matériel à la surface $\mathrm{du}$ sol ou en subsurface; et (2), les processus diagénétiques, qui interviennent après l'enfouissement des vestiges. Parmi ces derniers, les mouvements du sol (processus bio-pédologiques) et les phénomènes d'altération sont traités ici séparément. Dans tous les cas, les transformations physiques ou chimiques peuvent être plus ou moins dépendantes de facteurs biologiques, soit parce que la faune du sol et la flore interviennent sur le déplacement du sédiment, soit parce qu'elles contrôlent en partie la dissolution et la précipitation des minéraux.

\section{Processus sédimentaires}

La nature et l'intensité des transformations d'origine sédimentaire sont très variables, en fonction à la fois du type de contexte environnemental et de la rapidité de l'enfouissement, qui soustrait progressivement le niveau archéologique à l'action des processus géomorphologiques superficiels. Ces transformations peuvent aller de simples réajustements locaux à une érosion et à une redistribution complète des vestiges. Les sites se trouvent dans la majorité des cas dans un état de dégradation intermédiaire entre ces deux pôles (figure 2). D'une manière générale, deux grandes catégories de contextes, dont les implications sur la formation des niveaux archéologiques sont fondamentalement différentes, peuvent être distinguées : (1) les contextes d'accumulation pure; (2) les contextes d'accumulation - transit des sédiments. 


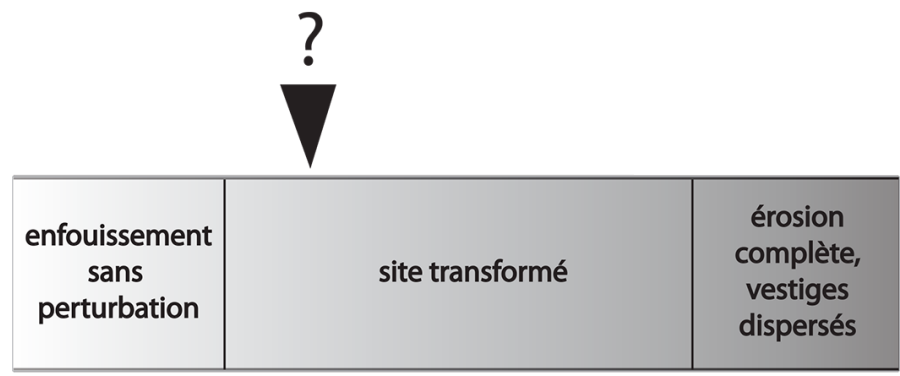

Figure 2 - L'un des principaux objectifs de l'approche taphonomique : déterminer à quel degré un site a été modifié par les processus naturels après son abandon par les hommes.

\subsection{Les contextes d'accumulation pure}

Les contextes d'accumulation pure correspondent à des environnements où les apports successifs de matériaux se superposent sans remaniement des dépôts antérieurs, l'accrétion sédimentaire pouvant alors se réaliser sans troncature significative. Ce type de situation, assez exceptionnel pour les sites paléolithiques, concerne surtout les plaines d'inondation des rivières (ex. niveaux magdaléniens de Pincevent), les environnements d'estuaires et de deltas vaseux, les parties lagunaires internes, les lacs et certaines zones d'accumulation éolienne, notamment lœssiques (ex. plaines du nordouest de l'Europe). Chaque niveau archéologique matérialise une paléosurface enfouie par les apports; la « résolution chronologique »d'un niveau est fonction de la fréquence relative des occupations et des apports de sédiments. La fréquentation par des groupes humains successifs d'un même lieu soumis à une accrétion sédimentaire faible ou nulle conduit à la formation d'un « palimpseste », terme proposé pour désigner un niveau archéologique constitué par l'imbrication de plusieurs occupations diachroniques [15, 16]. En géologie, le terme consacré est celui « d'enregistrement (ou de niveau) condensé ». Dans les contextes d'accumulation sédimentaire pure, la configuration initiale, telle qu'on peut l'appréhender à partir des plans d'objets, et l'intégrité des niveaux archéologiques sont souvent bien préservés malgré l'efficience de certains processus bio-pédologiques.

\subsection{Les contextes d'accumulation - transit des sédiments}

Les contextes d'accumulation - transit des sédiments, sont marqués par une redistribution des matériaux déjà déposés, qui contribuent pour une part plus ou moins importante à la sédimentation. Ces contextes se caractérisent par des processus de remaniements, avec des possibilités de troncatures dans les enregistrements archéologiques. Au cours de chaque épisode de sédimentation, des zones d'accumulation transitoire se forment (stockage temporaire); ces matériaux sont ensuite partiellement repris lors des épisodes ultérieurs et l'accrétion progressive des dépôts reflète le bilan global accumulation / érosion. C'est le cas très général des milieux de versant, des milieux éoliens où la déflation joue un rôle important (ex. pavages en milieu aride ou littoral), et des milieux alluviaux de moyenne à haute énergie. Dans les contextes d'accumulation - transit, les objets archéologiques, à l'instar du matériel détritique, 
subissent eux aussi une redistribution et leur organisation finale résulte à la fois de la configuration initiale d'origine anthropique et de la dynamique sédimentaire. L'intensité de la redistribution varie en fonction des processus impliqués et du temps d'exposition à ces processus. Cette redistribution peut être très rapide, par exemple en liaison avec des écoulements [17] et des mouvements en masse (glissements, coulées de débris), ou bien résulter de l'addition répétée de petits mouvements élémentaires ou d'une déformation lente du sol (reptation). Elle se caractérise par une translation des objets vers l'aval à laquelle s'ajoute généralement un processus de diffusion spatiale. Sur des versants en contexte périglaciaire, la solifluxion peut ainsi enfouir des niveaux d'occupation et redistribuer efficacement les vestiges bien que les vitesses de déplacement soient très faibles, le plus souvent, comprises entre 1 et $10 \mathrm{~cm} \cdot \mathrm{an}^{-1}$ [18-22]. Dans certains cas, des concentrations secondaires d'objets (c'est-à-dire après un transport) peuvent apparaître, en particulier en milieu fluviatile [17] ou de ruissellement [23].

\section{Processus bio-pédologiques}

L'existence de mouvements des vestiges sous l'influence des facteurs bio-pédologiques qui interviennent au cours de leur enfouissement ou postérieurement est un fait bien établi en géoarchéologie [1], puisqu'observés dans différents contextes, aussi bien en site de plein-air [24-31], qu'en grotte ou abri sous roche [32, 33]. Suivant l'importance des transformations liées à différents mécanismes comme le fouissage [34-41], le piétinement par les hommes [42, 43] et les animaux [44, 45], les châblis [46-49], l'argiliturbation dans les sols riches en argiles gonflantes de type smectite [1, 50, 51], la cryoexpulsion et la cryoturbation dans les milieux périglaciaires [52-55], ou l'effet de tamis (autotamissage) dans les matériaux grossiers sans matrice interstitielle [22, 56], les paléosurfaces anthropisées sont plus ou moins fortement dégradées. Ces facteurs, naturels ou anthropiques, sont susceptibles d'induire non seulement des déplacements verticaux et horizontaux, mais aussi la réorientation des pièces ainsi que leur tri. Les perturbations conduisent à une diffusion isotrope des vestiges dans un volume plus ou moins important de sédiments et à l'affaiblissement consécutif des concentrations. Elles peuvent s'accompagner d'un tri vertical en fonction de la dimension et de la forme des objets. Différents exemples de sites paléolithiques affectés par l'argiliturbation sont décrits en détail dans la littérature. Le cas du site de Nadung'a 4, localisé dans un sol vertique en contexte de plaine alluviale tropicale (Kenya) est spectaculaire à cet égard. La distribution des remontages, les fabriques et la présence de grands slickensides (surfaces argileuses brillantes et striées témoignant de glissements entre les agrégats à l'intérieur du sol) montrent que la dispersion verticale importante du matériel archéologique, soit sur près d'un mètre d'épaisseur, résulte essentiellement des mouvements liés aux alternances de retrait des argiles en saison sèche et de gonflement en saison humide. Malgré l'importante dispersion des vestiges, l'étude a permis de confirmer leur association avec les restes d'un proboscidien [50].

La bioturbation représente l'un des facteurs de pédogenèse mais aussi de perturbation les plus importants, et l'un des plus rapides, qui peut intervenir avant, pendant et postérieurement à toute occupation humaine, aussi bien en plein air en contexte d'accumulation pure qu'en contexte de versant, d'abri sous roche ou de grotte $[39,41$, $57,58]$. Lorsque le taux de sédimentation est faible, l'enregistrement archéologique 

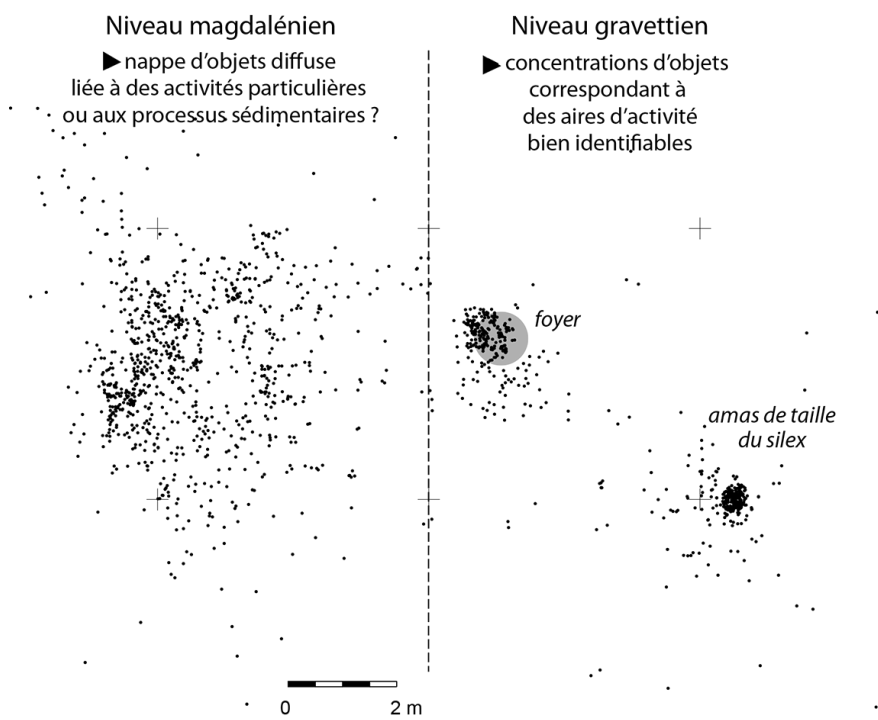

Figure 3 - Distribution des vestiges lithiques dans le site de Périgueux - rue Jules-Ferry (Detrain et al., non publié). Le niveau gravettien, qui présente une organisation aisément interprétable en termes d'aires d'activité juxtaposées, est interstratifié dans des alluvions limono-sableuses, tandis que le niveau magdalénien, qui se présente sous la forme d'une nappe d'objets sans concentration bien définie, provient de dépôts de versant. Dans ce dernier cas, l'étude taphonomique a pour but de tester l'hypothèse d'une redistribution du matériel lithique par la dynamique de versant.

correspond habituellement au mélange à des degrés divers de niveaux archéologiques initialement distincts [59]. L'enfouissement progressif des objets abandonnés sur le sol suite à l'accumulation de sédiments remontés en surface par l'activité biologique (déjections de vers de terre, termitières, déblais de mammifères fouisseurs) est un processus bien documenté et qui peut prendre une grande importance dans certains contextes, lorsque la sédimentation est faible, notamment en contexte tropical. Ce processus peut conduire à la formation de «pseudo-niveau archéologiques » en profondeur, suite à la concentration des objets à la base de la zone affectée par la bioturbation. C'est en particulier le cas des stone-lines [60, 61] et des industries associées [62-64].

\section{Taphonomie archéologique : objectifs, démarche et outils disponibles}

\subsection{Objectifs et évaluation des perturbations}

Les principales questions auxquelles l'étude taphonomique tente de répondre sont les suivantes : (1) dans quelle mesure l'organisation spatiale originelle (d'origine anthropique) du matériel archéologique a-t-elle été préservée (figure 3); (2) l'assemblage archéologique est-il homogène, c'est-à-dire provient-il éventuellement du mélange par les processus sédimentaires ou bio-pédologiques de niveaux initialement distincts (figure 4) ; (3) cet assemblage a-t-il conservé son intégrité, en d'autres termes, le matériel 


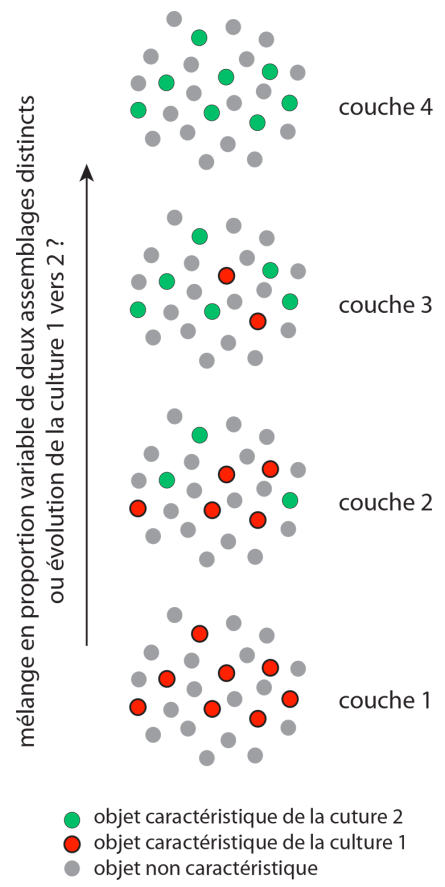

Figure 4 - Un problème usuel rencontré dans les sites paléolithiques stratifiés : comment distinguer des séries lithiques représentant l'évolution d'un technocomplexe vers un autre de séries lithiques mélangées par les processus taphonomiques.

récolté est-il un échantillonnage représentatif de l'assemblage initial ou ne correspondil qu'à une fraction particulière de cet assemblage en raison d'un tri sédimentaire (fonction de la dimension ou de la forme des vestiges) ou d'une altération différentielle (selon la composition et la taille des vestiges) ; enfin, (4) les artefacts présentent-ils un état de préservation individuel autorisant l'identification de leurs modalités de fabrication et d'utilisation?

La démarche de l'étude taphonomique comprend habituellement plusieurs étapes [23, 65] :

1. Dans un premier temps, les mécanismes sédimentaires et diagénétiques susceptibles d'avoir formé les couches dans lesquelles sont enfouis les vestiges sont déterminés. Cette étape permet de faire un certain nombre de suppositions sur les transformations qui ont pu affecter le niveau archéologique et de proposer des tests adéquats pour les mettre en évidence. Sur pente nulle, lorsque le taux de sédimentation est élevé et que les écoulements sont de faible énergie comme dans certains environnements de plaine alluviale ou dans les milieux lacustres, cette analyse « contextuelle » peut suffire à diagnostiquer un bon état de préservation du site. Néanmoins, une grande prudence s'impose, comme le montre l'exemple du site paléolithique moyen de Shi'Bat Dihya 1 (Yémen) interstratifié dans des dépôts limoneux de débordement d'une plaine alluviale [66]. Dans 

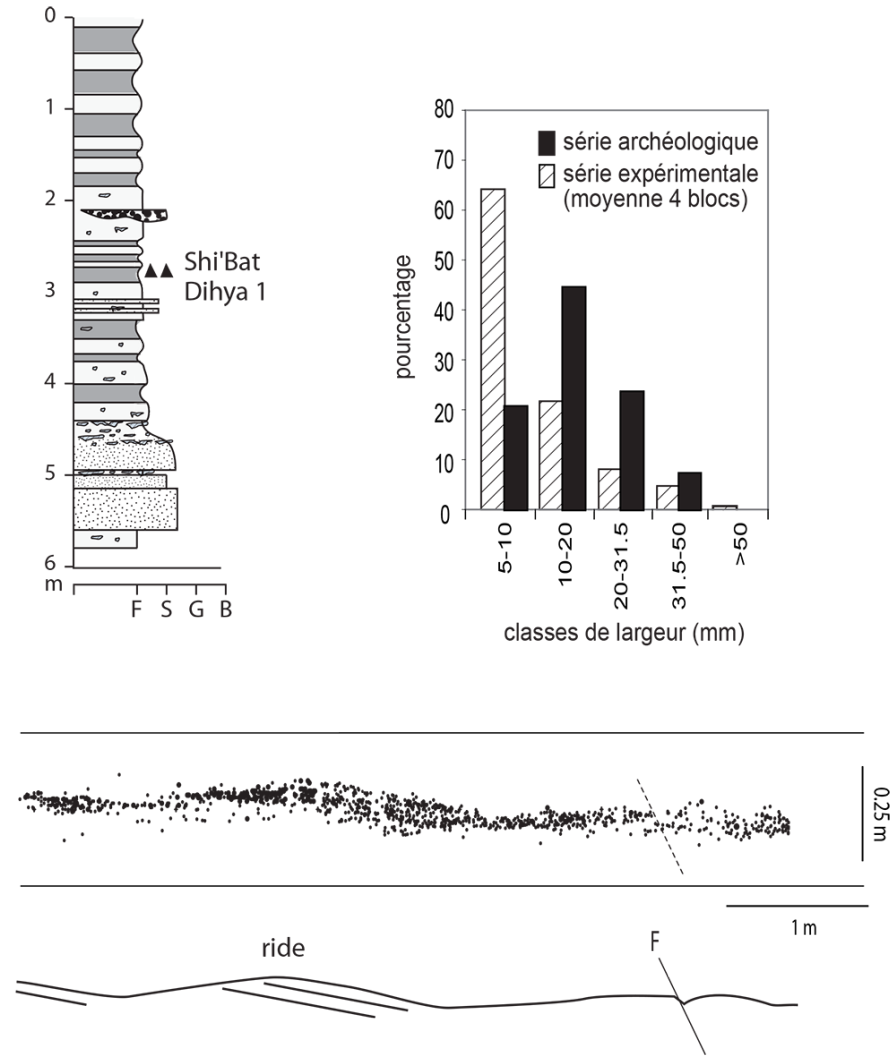

Figure 5 - Stratigraphie du site paléolithique moyen de Shi Bat Dihya 1 au Yémen, composition granulométrique du matériel lithique et projection verticale des objets, d'après Sitzia et al. (2012). Le niveau archéologique est interstratifié dans des dépôts de débordement de la rivière et a été trié par les écoulements. La série lithique présente un déficit à la fois en petits éléments et en grosses pièces par rapport aux séries expérimentales; le matériel s'organise en lits épousant des rides de courant.

cet exemple, l'étude a en effet démontré qu'en dépit du contexte relativement favorable car associé à des écoulements de faible énergie, les vestiges avaient été transportés, triés et réorganisés en rides de courant (figure 5). Il est possible que la présence d'amas de vestiges lithiques ait elle-même créé les conditions hydrodynamiques nécessaires au développement des rides de courant, en provoquant une diminution de l'épaisseur de la lame d'eau et une augmentation locale de la vitesse du courant.

2. Dans un second temps, on s'attache à rechercher, parmi les différentes caractéristiques du niveau archéologique, celles qui doivent être attribuées à la dynamique naturelle. Cette évaluation fait appel à des critères issus de la géomorphologie et consiste à comparer l'organisation et l'état de surface des vestiges à ceux qui ont été décrits dans des milieux sédimentaires naturels. Les critères pris en compte sont l'organisation spatiale des vestiges (《structures sédimentaires évidentes »), leur état de surface, leur disposition (fabrique) et 
leur granulométrie. Le diagnostic est bien entendu tributaire de la qualité des référentiels actuellement disponibles. À cette évaluation, s'ajoute l'appréciation du caractère « géologiquement in situ» du niveau archéologique, c'est-à-dire sa localisation dans des dépôts dont l'âge est compatible avec celui du matériel. Cet aspect reste cependant souvent difficile à appréhender et concerne surtout des contextes sédimentaires et des périodes pour lesquels à la fois une chronostratigraphie détaillée est disponible (par exemple pour les loess de Belgique ou du nord de la France $[67,68]$ ) et les technocomplexes successifs sont bien calés dans le temps (cas du Paléolithique supérieur européen). Les dates numériques $\left({ }^{14} \mathrm{C}, \mathrm{OSL}, \mathrm{TL}, \mathrm{U} / \mathrm{TH}, \mathrm{ESR}\right)$ peuvent concourir à préciser la contemporanéité des vestiges et des dépôts ; elles peuvent également mettre en évidence d'éventuelles inversions d'âge et, par conséquent, des perturbations potentielles de la stratigraphie.

3. Parallèlement, l'étude archéologique sensu stricto, basées sur l'étude typotechnologique et sur la distribution spatiale des raccords entre pièces, permet d'introduire une dimension de temps court dans l'étude des sites paléolithiques et de tester la cohérence des assemblages. Elle s'appuie sur la notion théorique de « chaîne opératoire » [69], placée dans un cadre spatio-temporel défini, et sur la mise en évidence de systèmes techniques de production. Du point de vue méthodologique, la multiplication des référentiels expérimentaux permet de valider la pertinence des propositions d'interprétation des séries lithiques, tandis que les raccords sur cassure d'objets et les remontages de produits provenant d'un même bloc de matière première permettent d'établir des relations de stricte contemporanéité entre vestiges [70]. L'analyse de la distribution spatiale (à la fois horizontale et verticale) des objets diagnostics ou appartenant à des ensembles de pièces raccordées, constitue l'outil central d'une reconstruction robuste d'ensembles archéologiques homogènes. Cette analyse est aujourd'hui grandement facilitée par l'exploitation numérique des données et la cotation systématique des objets.

Les différentes disciplines (archéologie, géoarchéologie, archéozoologie) doivent concourir à établir par leurs propres outils le degré de perturbation des ensembles qu'elles étudient relativement aux questions posées. Tous ces outils doivent être utilisés avec prudence, car ils n'apportent que des informations limitées qui peuvent parfois être interprétées de façon contradictoire. La confrontation des différentes approches permet de proposer un scénario plausible pour la formation du niveau archéologique, qui rende compte de l'ensemble de ses caractéristiques (figure 6). Ce scénario permet alors de répondre aux questions relatives à la distribution des vestiges, ainsi qu'à l'homogénéité et à l'intégrité $\mathrm{du} /$ des niveau(x) archéologique(s). Le consensus parfait est cependant rarement atteint, ce qui traduit la difficulté de reconnaître tous les processus qui ont affecté un site archéologique depuis sa création, jusqu'au moment de l'étude. 


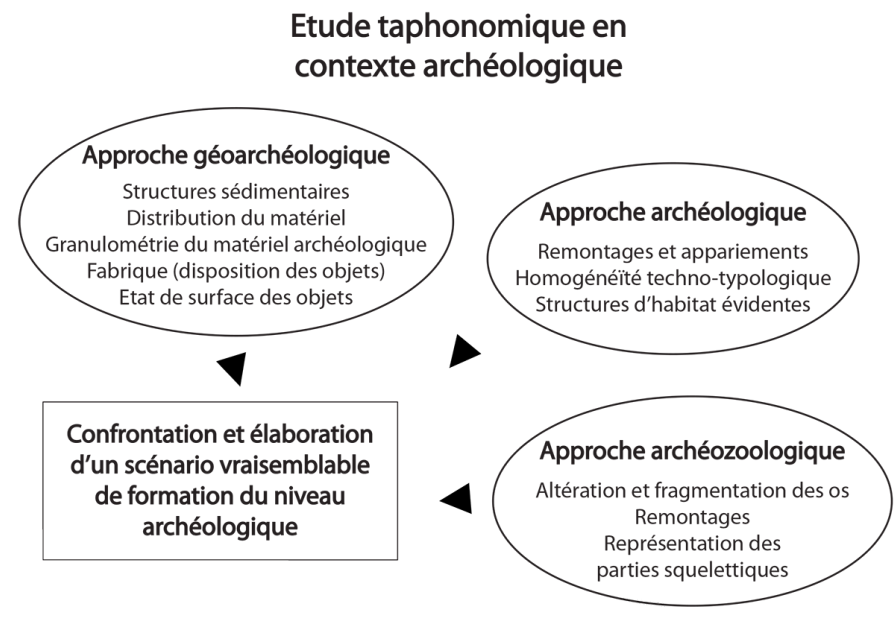

Figure 6 - La confrontation de différentes approches taphonomiques en archéologie concoure à l'élaboration d'un scénario robuste pour la formation du site.

\subsection{Le matériel archéologique comme signature anthropique... ou comme composante sédimentaire : quelques outils d'analyse}

\subsubsection{Structures sédimentaires}

La reconnaissance des 《structures sédimentaires évidentes » constitue une étape importante de l'analyse taphonomique. Pour le Paléolithique, les cas les plus fréquemment rencontrés sont (1) une association étroite entre le matériel archéologique et des lentilles ou des niveaux continus de graviers interstratifiés dans des matériaux plus fins, correspondant à des pavages d'érosion; (2) une déformation des niveaux archéologiques par divers processus (glissement, reptation, coulées, tris).

Une association étroite entre le matériel archéologique et un niveau de graviers interstratifiés dans des matériaux plus fins est fréquemment observée dans les sites paléolithiques français. Ces niveaux grossiers correspondent la plupart du temps à des pavages (lag deposits) mis en place par des écoulements en nappe ou chenalisés (rigoles, ravins) en contexte de ruissellement. Le matériel archéologique est généralement résidualisé par l'érosion et les éléments grossiers (nucléus, gros outillage...) atteignent des proportions élevées au sein des assemblages lithiques récoltés, en raison du transport préférentiel des éléments de petite taille. Les exemples de pavages contenant de riches assemblages archéologiques abondent en dehors de la zone lœessique nord-européenne, dans des environnements soumis au ruissellement où le bilan sédimentation / érosion est faible, en particulier en contexte de plateau ou de versant. C'est le cas assez général des sites du Paléolithique moyen en plein air dans le sud-ouest de la France. Le site de Combebrune 2 [71] localisé sur un plateau karstifié à proximité de Bergerac (Dordogne), est représentatif de nombreux sites de la région (figure 7). Le matériel archéologique dont l'âge va du Paléolithique moyen ancien (daté vers 190 ka, 


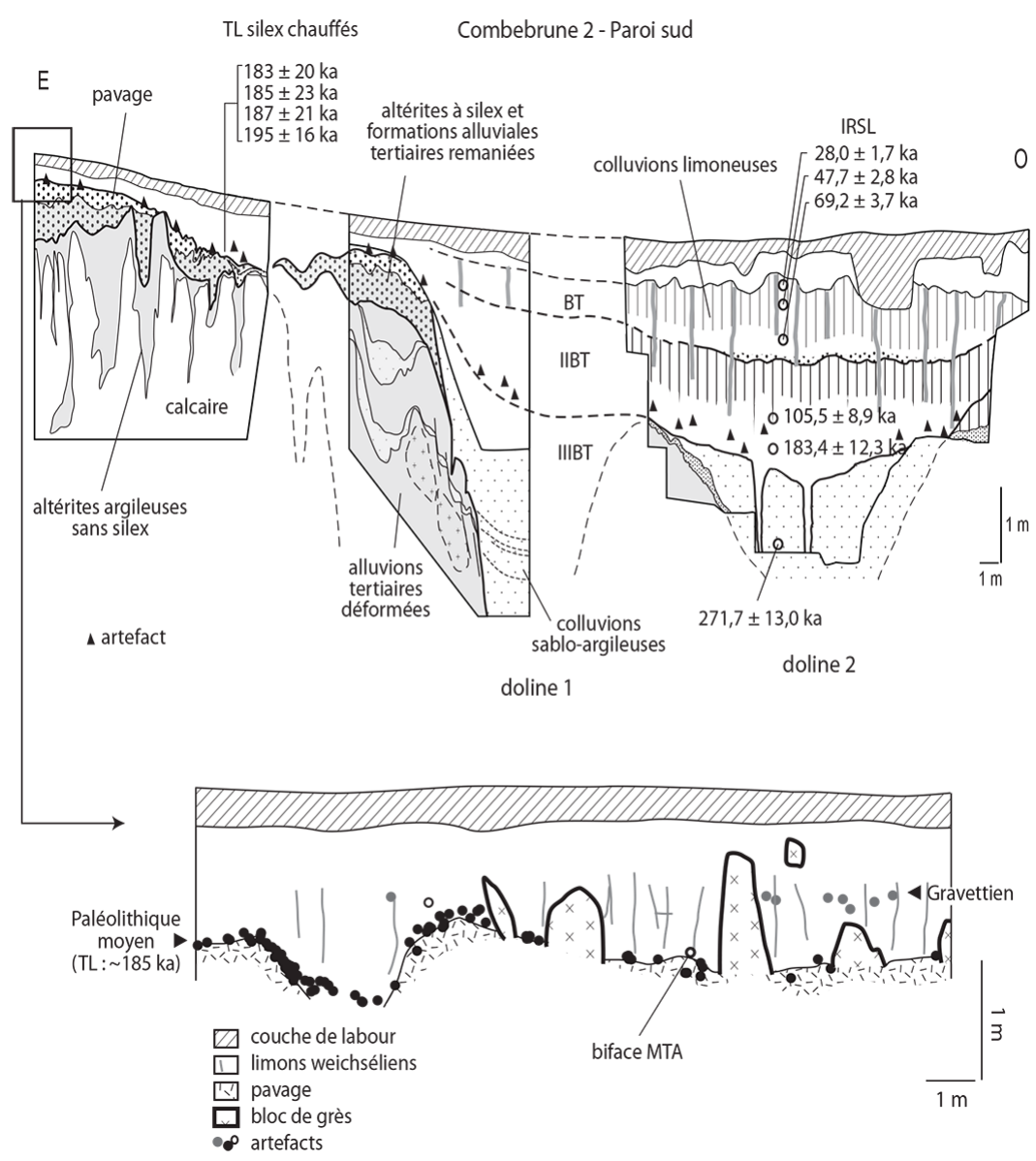

Figure 7 - Coupe du site de Combebrune 2 près de Bergerac, distribution du matériel archéologique et données chronologiques, d'après Frouin et al. (2014) modifié. Sur le plateau, le matériel archéologique forme un niveau reposant sur un pavage résiduel au sommet d'une couche d'altérites remaniées. Ce niveau, qui contient à la fois des pièces rapportées au Paléolithique moyen ancien ( $190 \mathrm{ka}$ ) et au MTA, est enfoui par des lœss weichseliens.

SIM 7-6) au Paléolithique moyen récent (MTA, SIM 3) est concentré dans un pavage composé de nodules de silex et de grès fragmentés en surface d'altérites argileuses à blocs. Ce pavage est recouvert par des limons éoliens déposés au cours du Pléniglaciaire supérieur. Dans un tel contexte, des occupations d'âge très différent peuvent être concentrées dans un même niveau résiduel (enregistrement condensé). Dans la mesure où les pavages sont le plus souvent mobiles (ils reflètent une concentration relative des éléments grossiers sur une surface soumise à l'érosion pendant une durée relativement longue; ces éléments sont néanmoins affectés par un mouvement de reptation plus ou moins rapide) et sont souvent déformés par les processus périglaciaires ultérieurs, l'organisation spatiale des occupations préhistoriques est généralement très perturbée. Ce phénomène de résidualisation explique que l'enregistrement archéologique puisse être à peu près continu alors même que l'enregistrement sédimentaire 


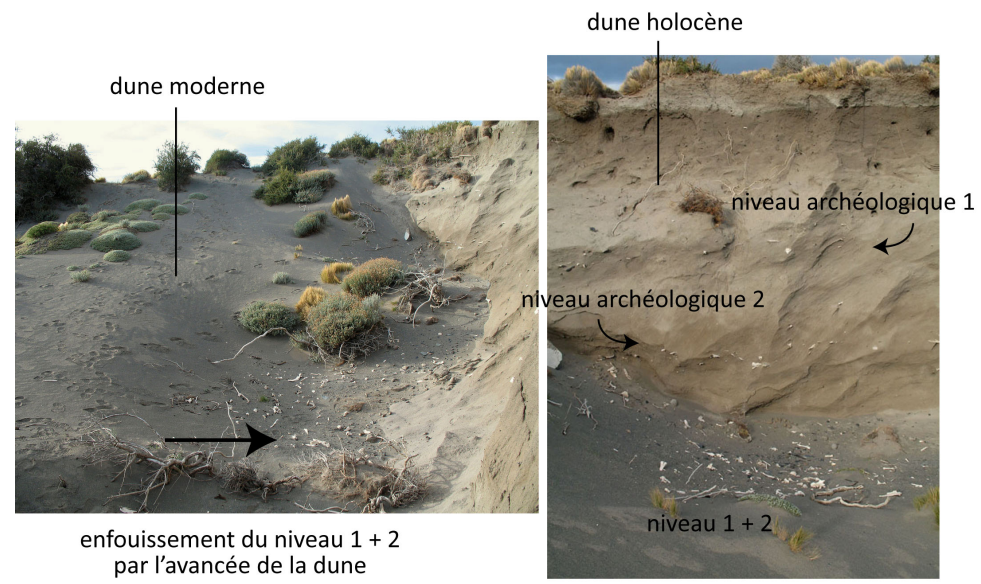

Figure 8 - Niveaux paléoindiens interstratifiés dans une dune holocène en bordure du Lago del Toro en Patagonie chilienne. La dune est en cours d'érosion et le matériel archéologique s'accumule au pied de l'escarpement où il forme un pavage. Ce pavage est progressivement enfoui par l'avancée de la dune moderne.

est très lacunaire, en raison de l'absence de dépôt pendant les interglaciaires et les interstadiaires.

Des phénomènes de pavage relativement similaires se produisent également en contexte fluviatile et éolien. On trouve, à travers toute l'Europe du Nord, de nombreux sites du Paléolithique inférieur dont les vestiges lithiques sont enfouis dans des dépôts de chenaux fluviatiles sablo-graveleux (ex. gisements acheuléens des terrasses de la Somme). Les configurations de type pavage alluvial sont fréquentes dans de nombreuses régions du monde, notamment en Afrique. En contexte fluviatile, le matériel grossier se concentre au fond des chenaux où le courant est le plus rapide. Lorsque la charge grossière naturellement transportée par la rivière est peu abondante, en raison de l'éloignement des reliefs ou d'une dynamique de versant peu active (en particulier dans certains milieux tropicaux), l'essentiel des éléments qui forment le pavage en fond de chenal correspond à du matériel archéologique provenant de l'érosion des berges. De nombreux sites du Paléolithique inférieur et moyen africain semblent être dans ce cas ; ces sites se caractérisent par des nappes de matériel de très grande dimension, composé en particulier de bifaces ou de galets aménagés, qui présentent souvent une disposition imbriquée [17, 72], ou bien par des accumulations localisées de matériel transporté et fortement triés par les écoulements [72]. Des exemples ont également été décrits en Israël [73] et en Italie [74].

En contexte éolien, la constitution de pavages (regs, desert pavements) est un phénomène très répandu [75] et les surfaces sur lesquelles sont mélangées des industries d'âge varié couvrent de grandes étendues au Sahara, au Chili (Atacama), en Arizona et en Australie. Les regs allochtones (ou alluviaux) correspondent à un épandage d'alluvions dont les éléments grossiers ont été concentrés en surface par le vannage éolien (résidualisation). Les regs autochtones sont dus à une météorisation in situ (fragmentation et altération) de la roche en place. Lorsque le vent y dépose des limons ou des 
sables, ces derniers migrent progressivement vers le bas par effet de tamisage et favorisent ainsi la formation d'un pavage de surface à vestiges archéologiques. La figure 8 illustre un exemple de genèse d'un pavage archéologique en contexte dunaire sur la marge d'un lac glaciaire de Patagonie chilienne.

Des déformations affectant les niveaux archéologiques, liées à des processus de versant (glissement de terrain - figure 9) ou des phénomènes périglaciaires (coulées, cryoturbation...) sont également communément observées dans le Paléolithique français. Beaucoup de sites ont en effet été exposés à des climats périglaciaires et ont été affectés soit par la solifluxion, c'est-à-dire une lente reptation du sol sous l'action des cycles de gel-dégel, soit par la cryoturbation sur terrain plat, souvent accompagnées d'un tri (sols polygonaux triés, sols striés). Le niveau solutréen du site de Cantalouette 2 localisé sur le flanc d'une doline près de Bergerac, où un amas de façonnage de pièces bifaciales a donné naissance à une coulée de solifluxion à front pierreux, est illustré sur la figure 10. Il est probable que la présence d'un amas de débris de silex sur le versant, en modifiant localement la susceptibilité du sol au gel et sa vitesse de reptation, ait entrainé la formation d'un front de coulée aux dépens d'une solifluxion en nappe plus régulière. Les sites du Paléolithique moyen de Saint-Amand-les-Eaux (Nord) et châtelperronien de Canaule 2 (Dordogne) sont représentatifs des nombreux sites paléolithiques affectés par des polygones triés (figures 11, 12) [54, 76-79]. La répartition chronoculturelle des sites portant la trace de figurés triés, visibles soit à partir des plans de répartition des objets, soit à partir des coupes de terrain, indique que ces phénomènes concernent le Paléolithique moyen [76, 80] et une grande partie du Paléolithique supérieur. Il semble notamment que ce soit le cas général pour le Gravettien et le Solutréen, contemporains du Pléniglaciaire supérieur. À l'opposé, les sites du Magdalénien final du Bassin parisien, comme Pincevent ou Etiolles, qui sont postérieurs aux périodes les plus froides du Pléistocène, sont comparativement bien mieux préservés.

Pour de nombreux sites paléolithiques, l'organisation des vestiges se caractérise cependant par une distribution en nappe plus ou moins dense, sans concentration d'objets identifiable que l'on puisse interpréter en termes de structure archéologique ou de figure sédimentaire. Dans de nombreux cas, ce type de configuration semble résulter d'une diffusion des vestiges sous l'action des processus géomorphologiques superficiels [21, 81]. Différents processus peuvent être impliqués dans cette diffusion, qui peut s'accompagner d'un déplacement latéral important lorsque la pente dépasse quelques degrés. Il s'agit en particulier de l'activité biologique [34], du ruissellement [23] et de la reptation du sol, notamment en contexte périglaciaire [18-22]. Deux modèles de translation vers l'aval et de diffusion susceptibles de donner naissance à des nappes de vestiges sont illustrés sur la figure 13. 


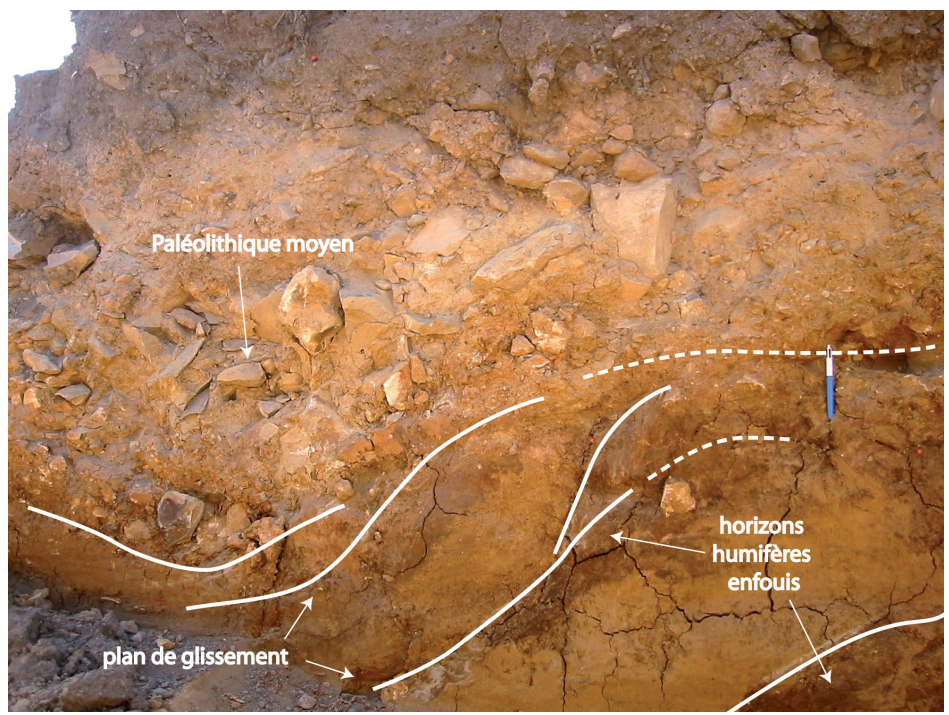

Figure 9 - Coupe du site paléolithique moyen des Leches (Dordogne). Le matériel archéologique a été déplacé par un glissement de terrain au sein des argiles altéritiques sous-jacentes.
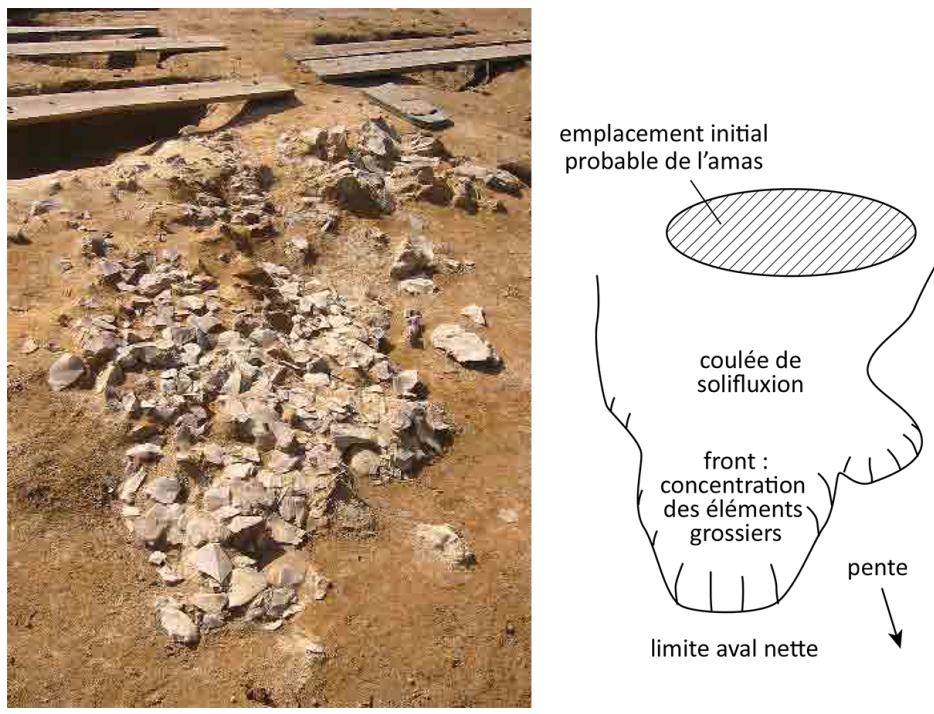

Figure 10 - Amas de taille du silex provenant du niveau solutréen de Cantalouette (Dordogne), d'après Lenoble et al. (2008) modifié. L'amas a été étiré sur la pente par la solifluxion. 


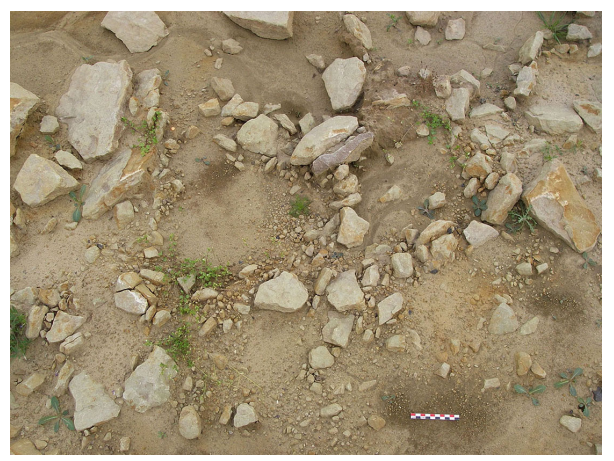

Figure 11 - Petits polygones triés sur le site paléolithique moyen de Saint-Amand-les-Eaux (Nord) (cliché Ph. Feray).

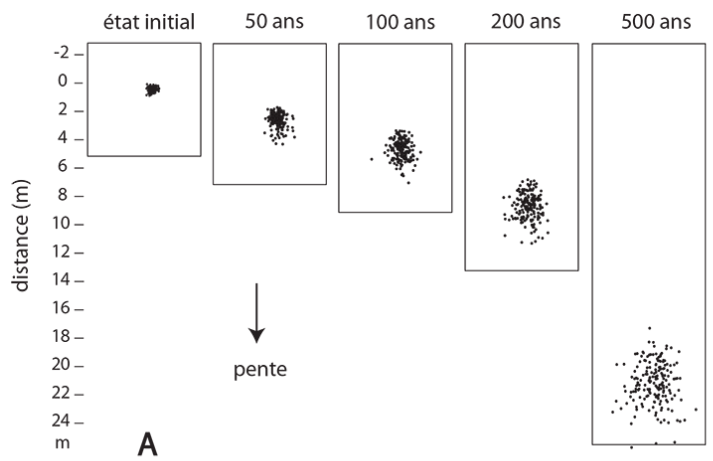

état initial
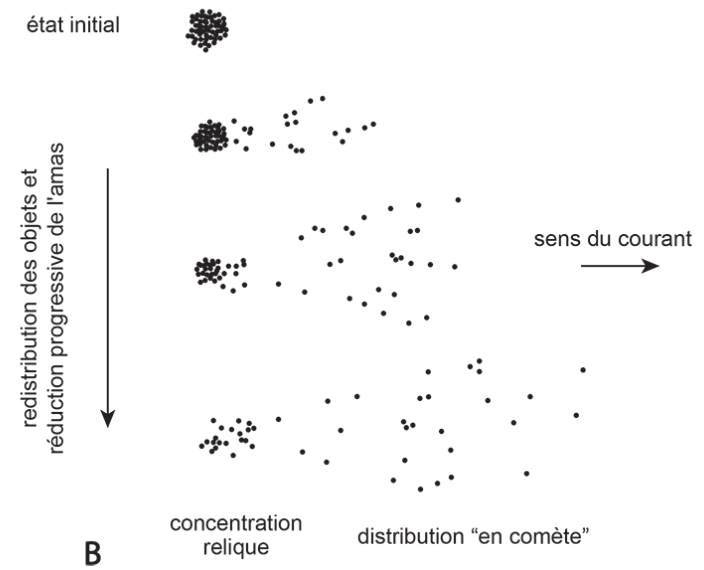

Figure 12 - Deux processus conduisant à la formation de nappes d'objets à partir d'amas lithiques. (A) redistribution du matériel par la solifluxion, d'après Lenoble et al. (2008); (B) ruissellement, d'après Lenoble (2005). 


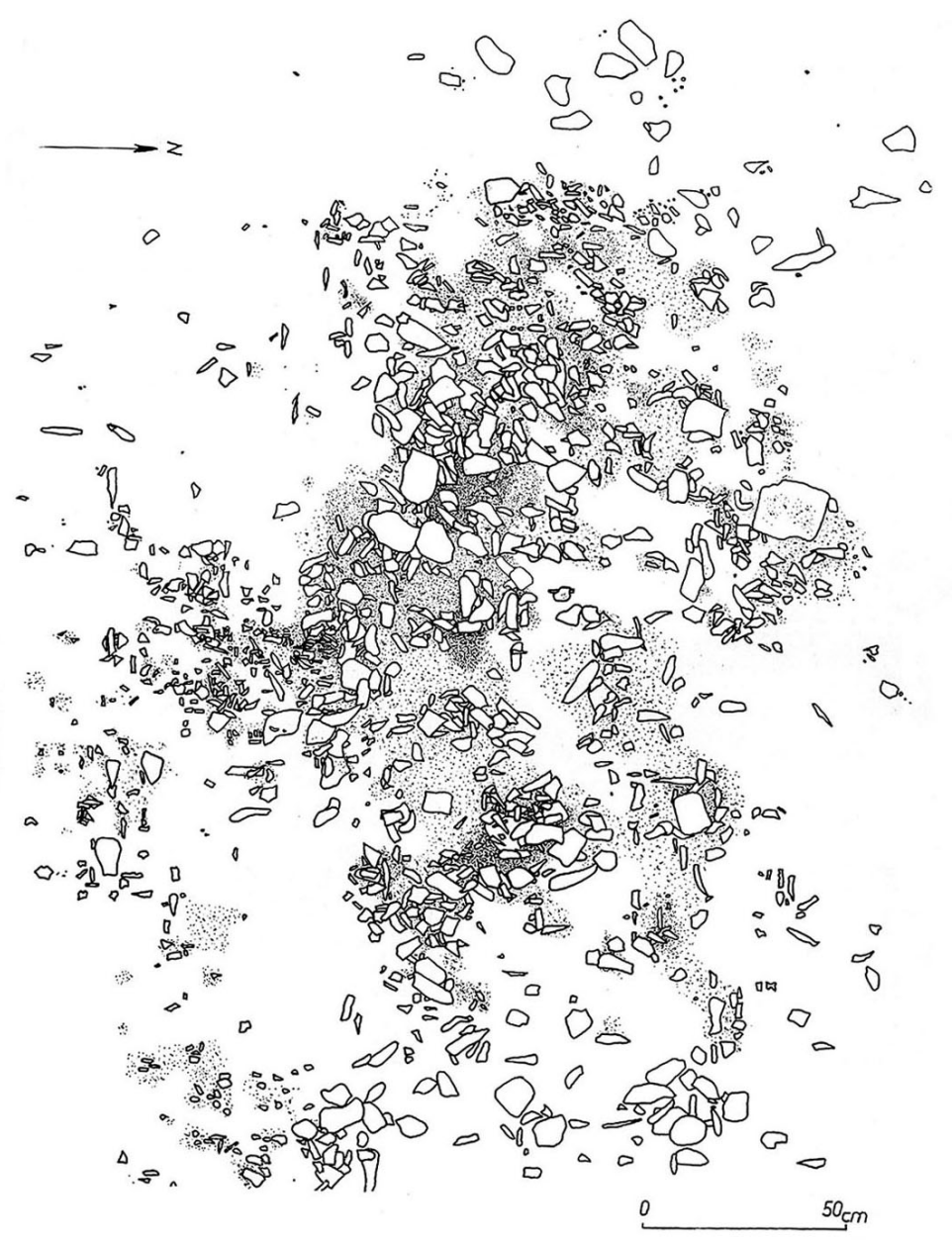

Figure 13 - Plan du site châtelperronien de Canaule 2, d'après Bordes (1972). Le matériel archéologique dessine des traînées qui correspondent aux cloisons de polygones triés typiques d'un environnement périglaciaire. 


\subsubsection{Fabriques}

L'étude des fabriques, c'est-à-dire l'analyse de l'orientation et de l'inclinaison des objets (matériel lithique ou fragments osseux) au sein des niveaux archéologiques, est souvent très informative. Ce type d'étude a initialement été développé dans le but de comprendre la formation des sites du Paléolithique ancien en contexte alluvial en Afrique et au Proche-Orient [17, 82-84]. Pour les dépôts de versant, l'application à l'archéologie est plus récente [85, 87]. Cet outil a fait l'objet d'importants développements dans les dernières décennies concernant à la fois la méthodologie, les types de sites investigués et l'établissement d'un référentiel en milieu naturel et expérimental [88-94]. Généralement, seul l'axe d'allongement L des objets (avec L > 2l, l étant la largeur) est considéré dans l'analyse. La fabrique est quantifiée par la mesure sur un échantillon représentatif d'une cinquantaine d'éléments. Le traitement des données fait appel à la statistique circulaire $[95,96]$ et au calcul de paramètres permettant de caractériser l'orientation moyenne (ex. vecteur moyen, concentration, variance circulaire), l'intensité de l'orientation préférentielle des vestiges, le type de distribution des orientations [97, 98]. La caractérisation statistique des séries testées est complétée par l'établissement des indices d'isotropie et d'élongation [99], fondés sur le rapport des vecteurs propres normalisés. Ces indices permettent de reporter la fabrique de chaque série testée sur un diagramme triangulaire où elle peut être confrontée à la fabrique de dépôts actuels ou fossiles [88].

Le type de fabrique permet de mettre clairement en évidence certains processus de perturbation et de sédimentogenèse. Les modifications de la disposition originelle des objets dans un niveau archéologique s'accompagnent en effet soit d'un accroissement de l'isotropie de la fabrique (disposition des objets plus désordonnée, en particulier dans le cas de la bioturbation ou de l'argiliturbation, suite aux alternances de retrait et de gonflement des argiles), soit de l'apparition d'une orientation préférentielle parallèle à la pente (typique des mouvements en masse des objets tels que la solifluxion et les glissements de terrain, mais aussi du ruissellement sur pente supérieure à $20^{\circ}$ ). La figure 14, sur laquelle sont pointées les fabriques d'un grand nombre de sites paléolithiques français en fonction des indices d'isotropie et d'élongation, montre qu'au moins les deux tiers des mesures s'écartent de façon significative du pôle « fabrique planaire », caractéristique des sites bien préservés et des valeurs obtenues lors d'expérience de rejets aléatoires d'objets sur le sol. Ce seul critère 《fabrique » indique donc clairement le caractère très général des modifications d'ordre taphonomique subies par de nombreux sites paléolithiques. L'un des intérêts de l'analyse des fabriques réside dans la possibilité de travailler sur l'orientation des objets allongés mesurée à partir de plans de couches archéologiques ou de photographies, et de pouvoir ainsi réévaluer les résultats de fouilles anciennes [94].

Dans certains cas, les fabriques ne permettent pas de statuer sur l'intervention éventuelle de certains processus sédimentaires, comme le ruissellement sur pente faible ou modérée. Dans ce type de contexte, les fabriques obtenues sont peu différentes de celles de sites non redistribués par les écoulements. L'observation détaillée de lots de mesures réalisées sur des surfaces restreintes (de l'ordre d'un ou deux mètres carrés) permet parfois de mettre en évidence une fabrique axiale bimodale, comparable aux 


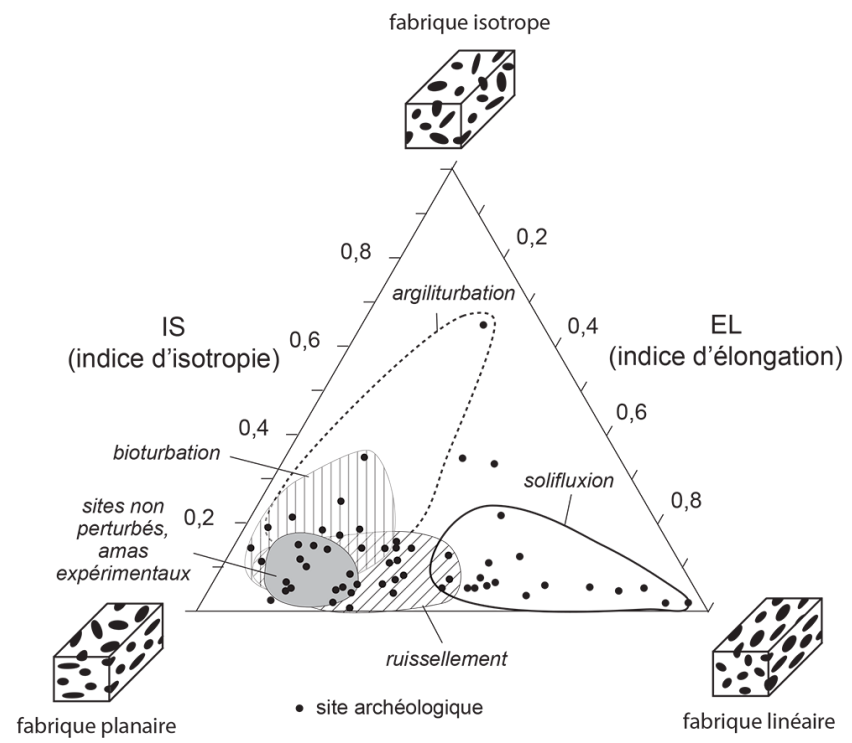

Figure 14 - Fabrique de niveaux paléolithiques français et aires correspondant à différents processus, d'après Bertran et Lenoble (2002).

fabriques typiques des milieux alluviaux. Elles restent cependant peu fréquentes, en raison de l'influence des irrégularités topographiques locales sur l'orientation des objets, et des interactions possibles entre les objets archéologiques et naturels (effets de blocage par exemple).

\subsubsection{Granulométrie du matériel lithique}

La granulométrie du matériel lithique est un outil qui a été initialement développé à des fins taphonomiques par Schick [17, 72], dans le but de comprendre le rôle de la dynamique alluviale dans la genèse des sites oldowayens d'Afrique de l'est. L'analyse consiste à séparer les différents constituants en classes dimensionnelles à l'aide de cribles ou de tamis. La gamme de taille de la fraction étudiée est variable selon les questions posées, la largeur des pièces étant la principale dimension considérée. Tous les éléments (nucléus, supports, éclats, outils, débris) sont dénombrés dans chaque refus de tamis [23, 100-103]. En pratique, seul le matériel de largeur supérieure à $7,1 \mathrm{~mm}$ (soit une maille $\mathrm{d}=5 \mathrm{~mm}$ ) est généralement pris en compte dans l'analyse, dans la mesure où cette maille est la plus petite utilisée en routine sur les fouilles pour récupérer le matériel lithique. La mise évidence d'un tri d'origine sédimentaire sur cette fraction peut par ailleurs avoir des conséquences importantes pour l'étude archéologique de la série.

Plusieurs mécanismes sédimentaires ont la capacité de transporter sélectivement les particules en fonction de leur taille. Dans le cas des écoulements naturels (ruissellement, rivières), les courbes granulométriques caractéristiques sont différentes en fonction des zones de résidualisation, de transit ou d'accumulation du matériel. Par conséquent, le tri granulométrique est donc une signature taphonomique qui peut être 


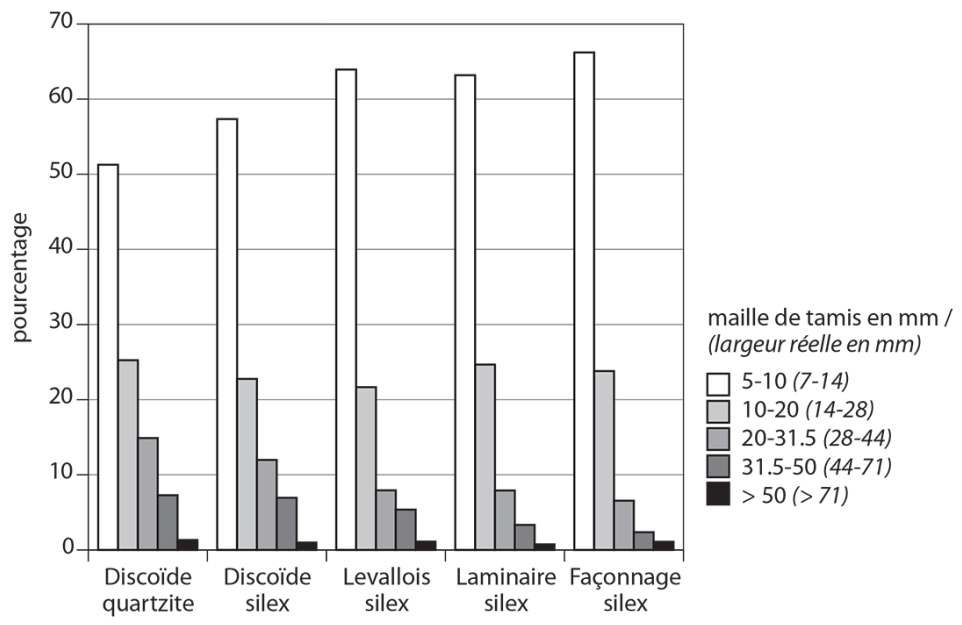

Figure 15 - Distribution granulométrique de séries expérimentales taillées selon des méthodes et à partir de matières premières différentes, d'après Bertran et al. (2012) modifié.

aisément mise en évidence par l'analyse de la composition d'une série lithique. Les expériences réalisées montrent que les produits issus du débitage de silex ou d'autres matières premières ont une composition granulométrique relativement constante, les différences de proportions (en nombre d'éléments) pour chaque classe granulométrique étant toujours inférieures à $15 \%$ d'un débitage à l'autre (figure 15). Les différences observées, bien que faibles, sont fonction de plusieurs facteurs, comme la matière première utilisée, la dimension des produits recherchés ou encore la maîtrise du tailleur. La proportion de fragments décroît rapidement des petites vers les grandes tailles, c'est-à-dire que le débitage (ou le façonnage) produit énormément de petits débris et peu de grandes pièces. La distribution suit approximativement une loi de type exponentielle décroissante ou de type Weibull [17, 23, 100-103].

Lorsque la distribution granulométrique d'un niveau archéologique dans lequel des activités de taille du silex ont eu lieu s'écarte sensiblement de la composition typique obtenue lors du débitage ou du façonnage, cela implique qu'un tri granulométrique des vestiges s'est produit après la taille. Le tri peut avoir plusieurs origines, soit anthropique et liée aux activités humaines qui ont présidé à la constitution de l'assemblage lithique abandonné sur le site, soit naturelle et provoquée par les processus sédimentaires qui ont affecté le site avant qu'il ne soit totalement enfoui. Dans la mesure où l'utilisation de la granulométrie numérale sur l'ensemble du matériel de largeur supérieure à 2,8 ou $7,1 \mathrm{~mm}$ permet en grande partie de s'affranchir de la variabilité d'origine anthropique (exportation-importation d'objets) qui concerne surtout les éléments de grande taille, la reconnaissance d'un tri dans une série suggère le plus souvent une modification du niveau archéologique par les processus sédimentaires.

La confrontation avec les informations fournies par les autres analyses permet généralement de proposer un scénario robuste des modifications qui sont intervenues. Le constat d'un tri a alors d'importantes implications sur la signification que l'on 

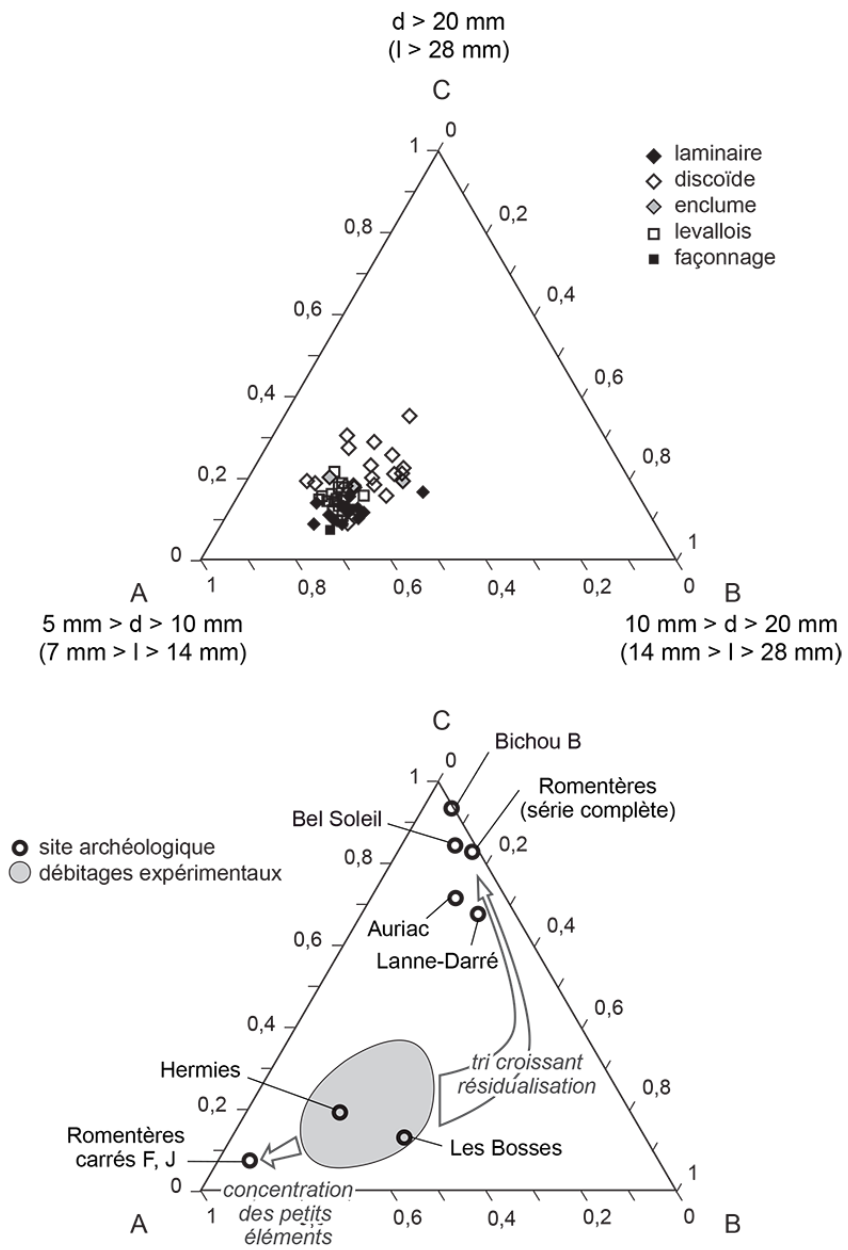

Figure 16 - Composition granulométrique de séries expérimentales et archéologiques (Paléolithique moyen et inférieur), d'après Bertran et al. (2012) modifié.

peut donner à la distribution spatiale, mais aussi à la composition techno-typologique de la série lithique. La figure 16, complétée d'après Bertran et al. [101], illustre la composition granulométrique d'un certain nombre de séries lithiques du Paléolithique inférieur et moyen. Si plusieurs se placent à proximité de l'aire attendue pour un site sur lequel des activités de taille du silex ont eu lieu, d'autres s'en écartent sensiblement et reflètent des assemblages triés par les écoulements, soit en contexte fluviatile, soit en contexte de versant (ruissellement). Ces assemblages se sont donc avérés être peu favorables à une étude techno-économique fiable, dans la mesure où leur intégrité n'a pas été conservée. On constate notamment que les outils de grande dimension et les nucléus sont largement surreprésentés par rapport aux outils sur éclat de petite dimension (figure 17). 

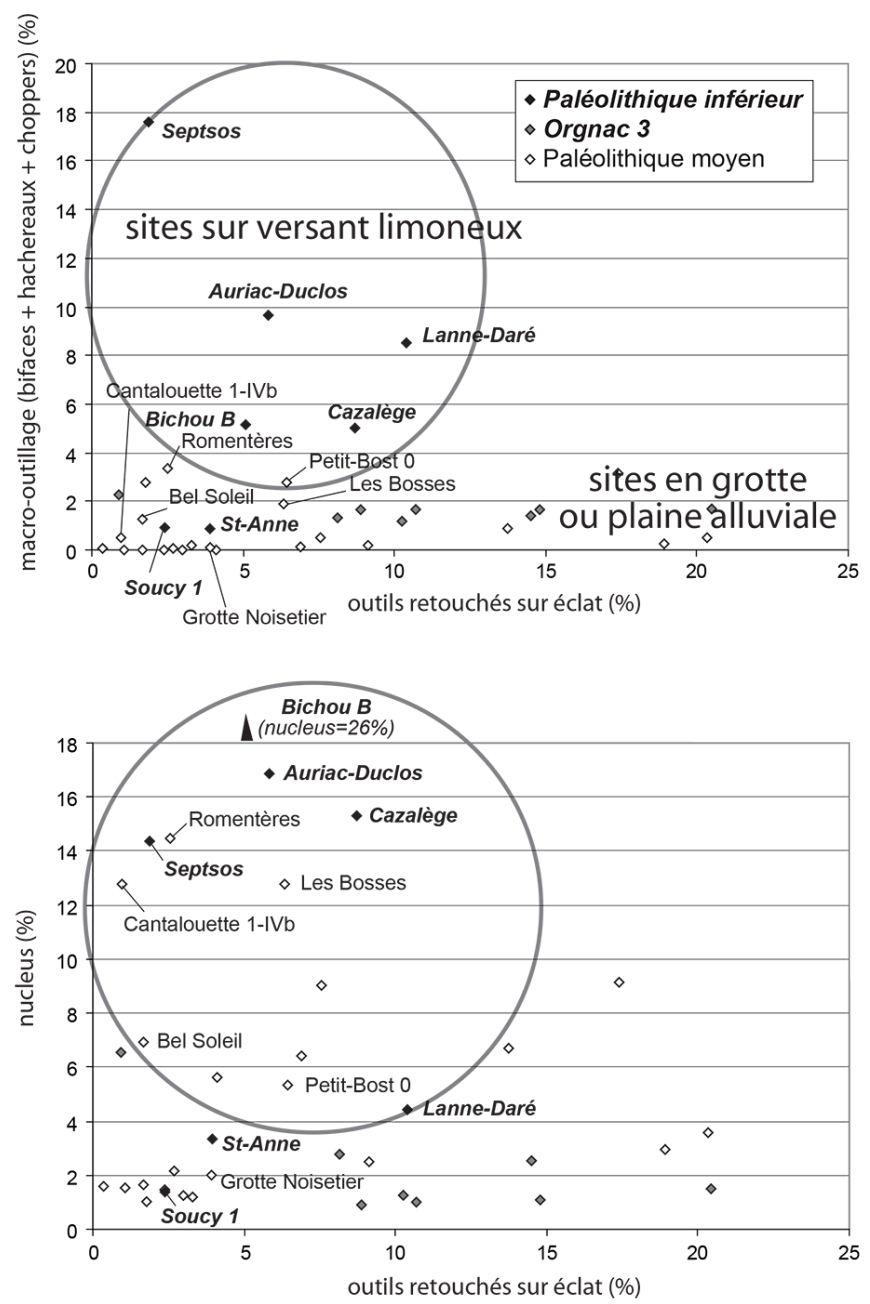

Figure 17 - Pourcentage de gros outillage et de nucléus en fonction de l'outillage de petite dimension (sur éclat) pour différentes séries du Paléolithique inférieur et moyen, complété d'après Bertran et al. (2012). L'analyse granulométrique des séries entourées par un cercle montre qu'elles ont été triées par des écoulements.

Lorsqu'on dispose de vastes surfaces fouillées, la cartographie de la distribution des objets en fonction de leur taille (regroupée en classes) peut apporter des arguments forts en faveur d'un tri granulométrique, notamment lorsque des gradients sont perceptibles. La figure 18 illustre le cas d'un site du Paléolithique moyen dans des limons colluvionnés du sud-ouest de la France, où la carte met clairement en évidence une concentration des vestiges les plus grossiers à l'amont du versant (zone résidualisée) et la présence de matériel de plus petite taille à l'aval (matériel redistribué par le ruissellement). 


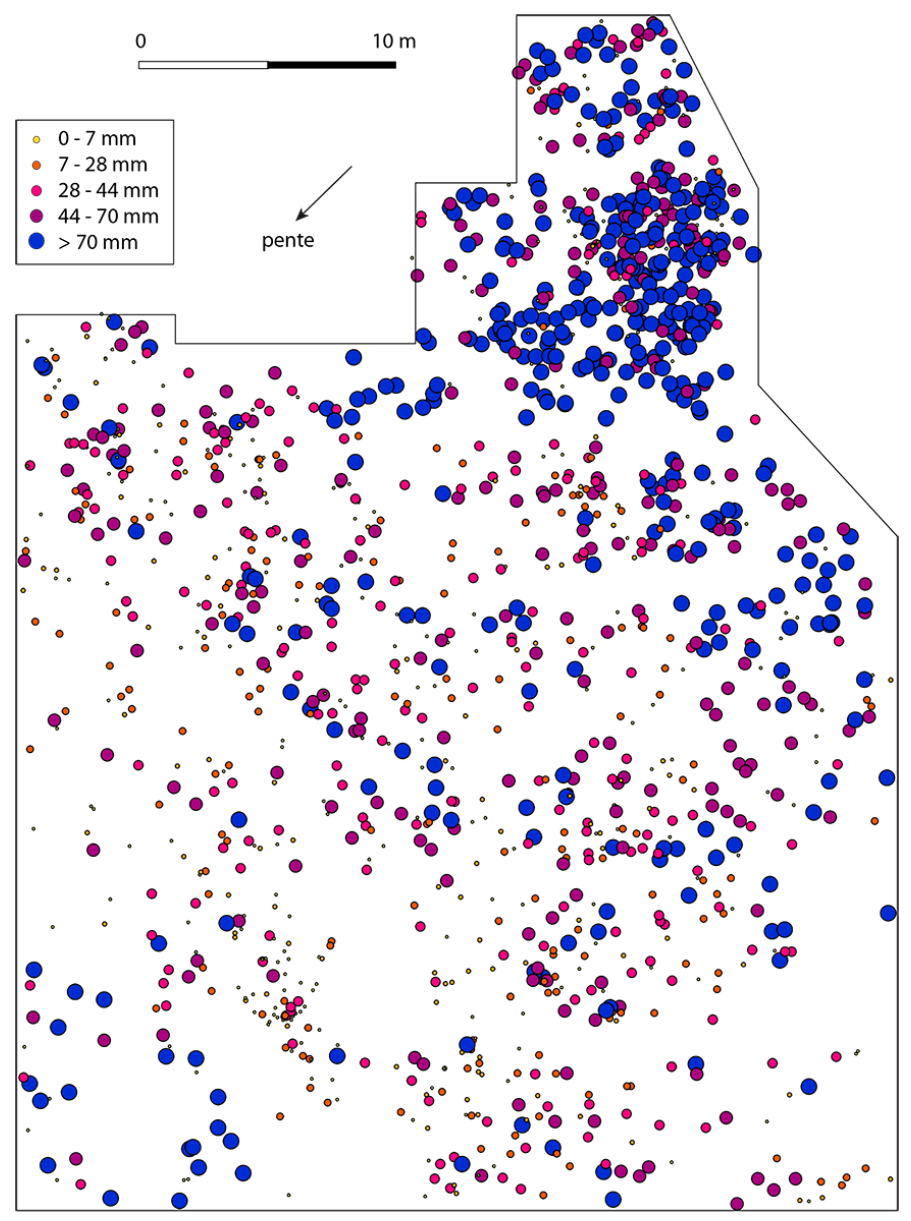

Figure 18 - Distribution des pièces lithiques sur le site de Romentères (Landes) classées en fonction de leur largeur. On distingue clairement un gradient granulométrique parallèle à la pente.

\subsubsection{Remontages}

Le remontage de pièces provenant d'une même matrice originelle (bloc, élément anatomique, etc.) permet la reconstitution fine des étapes de réduction de cette matrice. Cette méthode est connue depuis la fin du XIX ${ }^{\mathrm{e}}$ siècle, mais elle n'a longtemps été appliquée que de façon anecdotique et souvent dans un but technologique, à partir d'artefacts lithiques [104-109]. Dans ce cadre, et dès l'origine, elle s'est appuyée sur les données fournies par les débitages expérimentaux. Les avantages sont nombreux car le remontage permet d'introduire une relation chronologique de haute résolution entre des objets archéologiques, tout en ajoutant une dimension dynamique à la vision statique des niveaux archéologiques fournie par les analyses spatiales. La représentation spatiale des relations entre les objets provenant de la même matrice exprime le bilan des mouvements de ces objets, postérieurement à leur production [110]. Ces déplacements peuvent être d'origine anthropique (volontaire ou non) ou naturelle (fi- 


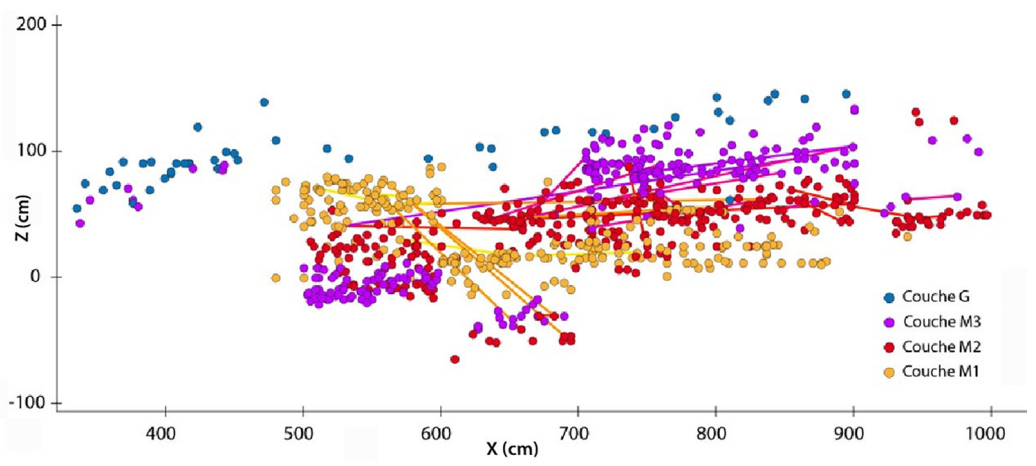

Figure 19 - Projection verticale des objets lithiques du Moustérien (niveaux M1 à M3) et de la base de l'Aurignacien (G), Caminade-est, fouilles Sonneville-Bordes (1958-66) puis Bordes et Lenoble (1999-2001). Les traits entre les points représentent des raccords. L'un des buts de l'analyse taphonomique est d'isoler des ensembles de vestiges montrant une cohérence chronoculturelle. Pour cela, elle doit démêler ce qui, devant une telle configuration, relève de comportements humains anciens (succession des occupations, déplacements et aires d'activités), de processus naturels post-dépositionnels (noter les nombreux raccords entre les niveaux M2 et M3 en X $=700$ à 900), ou encore d'erreurs de fouilles ou de transcription de l'information contenue dans les carnets de fouille, comme on le voit ici au niveau de la travée $\mathrm{X}=600$ à 700 .
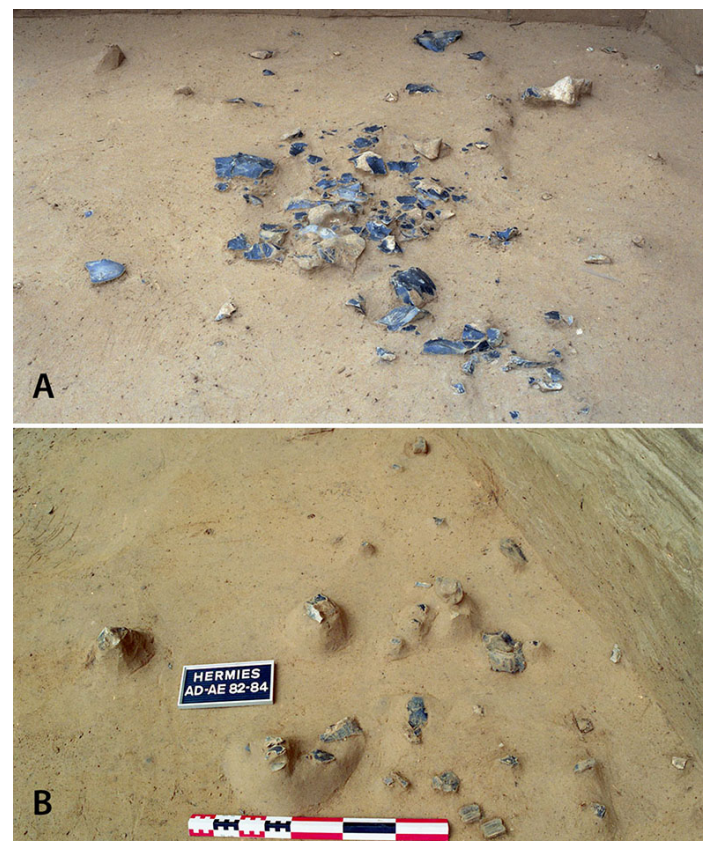

Figure 20 - Conservation différentielle des amas de débitage du site d'Hermies le Champ Bruquette (Pas-de-Calais). (a) concentration sur $1 \mathrm{~m}^{2}$ de 162 artefacts lithiques correspondant à trois séquences de réduction intégralement conservées; (b) groupement relatif de 65 artefacts disséminés sur $3 \mathrm{~m}^{2}$, dont 44 ont pu être raccordés en une seule et même séquence. 
gure 19, 20). Les raccords de cassure (réassemblage de fragments d'un même objet) sont plus délicats à interpréter car l'origine de la fragmentation (fracture au débitage, fracturation volontaire, choc accidentel, gélifraction, etc.) est rarement évidente; ils fournissent néanmoins des témoignages supplémentaires des dynamiques ayant affecté les niveaux archéologiques.

L'interprétation de la distribution des raccords, s'ils établissent des liens de contemporanéité forts entre les pièces, n'est pas sans écueils, principalement en raison de la difficulté d'apprécier sur des bases purement spatiales les relations chronologiques entre les ensembles clos que sont les ensembles remontés (voir le débat entre Bordes [111, 112] et Cahen et al. [113-115]. L'éventualité du « recyclage » des vestiges lithiques d'un groupe humain par leurs successeurs doit également être gardée en mémoire. De tels cas sont attestés dans les industries du Paléolithique moyen (indiqués parfois par une double patine) mais ils restent marginaux. L'analyse de la distribution des raccords à partir de projections verticales reste cependant l'outil le plus puissant pour démontrer l'invalidité d'une séquence archéologique [9, 27, 116-120], en permettant de tester si la dispersion des vestiges ne se produit qu'au sein de chaque niveau archéologique ou, à l'inverse, si des mélanges entre plusieurs niveaux sont intervenus. Leur analyse peut mettre en évidence des cooccurrences de vestiges causées par des déplacements secondaires et non par des changements graduels d'ordre technologique, et permet ainsi de réexaminer la question des éventuelles transitions culturelles (ex. de la transition Middle / Late Stone Age en Afrique [121, 122] ou de l'interstratification entre différents technocomplexes [9, 119]).

\subsubsection{Cohérence chrono-culturelle}

L'évaluation de la cohérence chrono-culturelle est principalement fondée sur l'étude techno-typologique des assemblages archéologiques. Le diagnostic dépend très étroitement de l'état de connaissance des différents techno-complexes et de la qualité de conservation et d'analyse des niveaux archéologiques qui servent de référence pour la définition de ces technocomplexes. Cet état des connaissances ne cesse donc d'évoluer au gré des nouvelles fouilles et de la réévaluation des anciennes séries. Ainsi, grâce à l'archéologie préventive, la multiplication des découvertes de niveaux archéologiques de plein-air correspondant à des occupations limitées dans le temps tend à démontrer qu'une grande partie des assemblages ayant servi à la définition des techno-complexes correspondent à des enregistrements condensés, à faible résolution chronologique et qui rassemblent donc des objets d'âge significativement différent. Par ailleurs, du fait de l'existence de types d'objets ou de chaines opératoires ayant une distribution chronologique très longue, la résolution que permet l'évaluation de la cohérence chronoculturelle peut être faible. C'est notamment le cas pour une grande partie du Paléolithique inférieur et moyen européen. L'appréciation de la cohérence chrono-culturelle (ou biostratigraphique) des vestiges qui composent un même ensemble archéologique est un mode d'analyse habituel, mais qui n'est donc pas sans difficultés. Les résultats obtenus à partir de ce type d'analyse doivent ainsi nécessairement être croisés avec d'autres types d'information. 


\subsubsection{Datations numériques}

Les dates numériques peuvent souvent être utilisées avec profit dans un but taphonomique. Il est en effet fréquent d'observer des âges radiocarbone incohérents au sein d'une séquence archéologique, soit parce qu'ils ne correspondent pas à l'âge attendu pour un technocomplexe donné, soit parce que les relations stratigraphiques entre les objets datés ne sont pas conformes aux âges obtenus (inversions stratigraphiques). Le problème reste cependant complexe dans le cas du radiocarbone, dans la mesure où de nombreux cas d'incohérence peuvent être liés à des problèmes de pollution des échantillons par de la matière organique plus récente [123, 124]. Avec le développement de méthodes capables d'éliminer toute pollution telle que l'ultrafiltration du collagène [124], l'identification d'inversions stratigraphiques ou d'outliers devient plus robuste et permet de mettre en évidence de possible phénomènes de remaniement et de mélange de matériel dans une séquence. La principale limitation à cette analyse réside dans la nécessité de s'assurer que le matériel daté n'a pas eu un comportement différent de celui du reste du matériel archéologique. Ce peut être typiquement le cas pour les fragments de charbon de bois, de petite taille et sensibles au transport par l'eau et le vent.

Pour les périodes au-delà de la limite d'application du radiocarbone, les méthodes de datation par luminescence apportent souvent un éclairage très précieux sur l'histoire taphonomique d'un site. L'utilisation conjointe de la thermoluminescence (TL) sur les silex ou les quartz taillés et de la luminescence stimulée optiquement (OSL) sur les sédiments dont proviennent les pièces archéologiques permet en effet d'évaluer leur contemporanéité, dans les limites de résolution de ces méthodes. Ce type d'analyse a été appliqué sur plusieurs sites du Paléolithique moyen ancien dans des colluvions lœessiques du sud-ouest de la France [125] et a montré sans ambiguïté l'association dans un même niveau de pièces d'âge très différent et pour certaines, d'un âge beaucoup plus ancien que celui du sédiment encaissant (figure 21). Ceci suggère que, dans une région où la densité des sites paléolithiques est élevée et où la sédimentation reste faible au cours du Pléistocène, ces phénomènes de remaniement et d'association au sein de pavages de pièces d'âge sensiblement différent pourraient être très répandus.

L'utilisation croisée de l'analyse taphonomique (degré de cohérence technologique, remontages) et de différentes méthodes de datation $\left({ }^{14} \mathrm{C}\right.$, OSL) a été appliquée sur un site paléoindien stratifié de plein-air au Brésil [126]. L'étude révèle la présence de deux niveaux d'occupation distincts, bien préservés, qui contrastent avec des ensembles davantage perturbés par l'action éolienne et la bioturbation, avec une dispersion des petits éléments lithiques. Dans le désert australien (New South Wales), Fanning et al. [127] montrent que la combinaison de datations OSL et ${ }^{14} \mathrm{C}$ permet de mettre en évidence l'âge composite des assemblages archéologiques, soulignant ainsi la diachronie des occupations et la variabilité spatiale des âges de surface dans les zones arides. Sur le site Folsom de Barger Gulch Locality B (Colorado), des datations ${ }^{14} \mathrm{C}$ sur charbon de bois et matière organique, couplées à l'analyse des distributions verticales d'artéfacts et aux données stratigraphiques, précisent la chronologie des évènements pédo-sédimentaires et les phases de dispersion des vestiges : enfouissement fini-pléistocène d'une composante Folsom sous des dépôts éoliens, érosion (surface de 
déflation), développement pédologique (horizon humifère), troncature partielle du sol (déflation), puis de nouveau enfouissement [128].

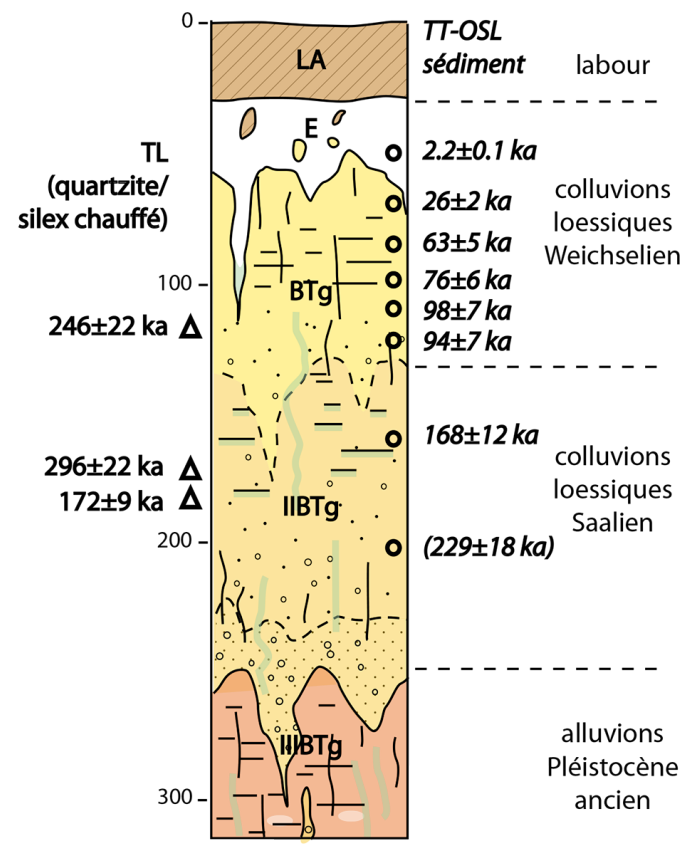

Figure 21 - Stratigraphie et chronologie du site de Romentères (Landes), d'après Hernandez et al. (2012) modifié. Une partie des pièces datées par TL a un âge significativement plus ancien que celui du sédiment encaissant. Le niveau archéologique inférieur correspond à un assemblage de pièces d'âge différent.

\section{5 État de surface du matériel lithique}

Les artefacts lithiques, notamment ceux en silex, montrent régulièrement des altérations ou des modifications postdépositionnelles des tranchants et des surfaces (PDSM : postdepositionnal surface modification) pouvant entraver leur reconnaissance technologique, typologique et fonctionnelle. Ces modifications peuvent affecter les traces d'utilisation, les détruire ou bien encore les imiter. D'ordre mécanique / tribologique (frottement), thermique ou chimique, elles peuvent prendre des formes variées, et se combiner à différentes échelles, en lien avec les processus sédimentaires et/ou diagénétiques, avant, pendant et après enfouissement : écaillage, fissurations, ébréchures, fractures, émoussé des bords et des arêtes, stries, patine (blanche / patination, brillante / lustre, bleue, vermiculé / dentritique...), spots brillants / polis plats, déformations plastiques, changement de couleur [45, 129-133]. Via l'étude des états de surface, il est donc possible, jusqu'à un certain point, de déduire les conditions paléoenvironnementales dans lesquelles les artéfacts lithiques ont évolué et les processus de formation des sites [134, 135] (figure 22). 


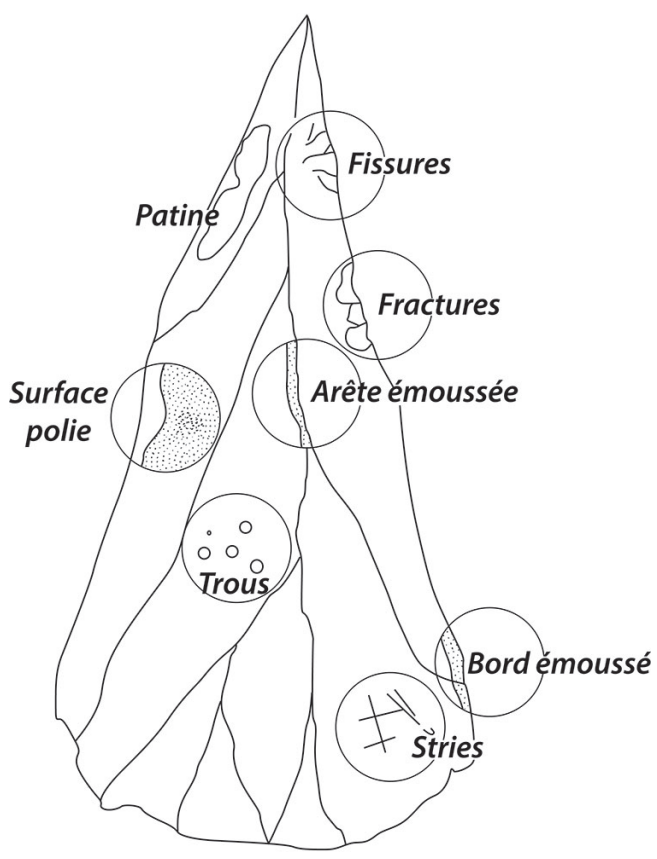

Figure 22 - Types d'altération d'un objet en silex, d'après Burroni et al. [132].

\subsection{Impact des conditions désertiques}

En contexte désertique, les éléments rocheux naturels et les vestiges lithiques subissent plusieurs types d'altération [129, 136-139], en lien direct avec le vent mais aussi en fonction des oscillations thermiques quotidiennes (thermoclastie), des conditions de salinité (haloclastie), d'humidité ambiante (brouillard) et du sol, et la végétation. Les particules transportées par le vent, en particulier les grains de quartz, agissent comme des micro-burins sur les éléments rocheux, notamment quand la végétation est absente (aeolian sandblasting). Cette météorisation mécanique, ou corrasion, a pour conséquence de façonner des surfaces luisantes, d'aspect gras (poli désertique), qui présentent au microscope un aspect dépoli. Stapert [129] décrit un lustre (sheen) sans couleur et d'apparence grasse (wind-gloss), qui peut être plus ou moins présent à la surface des silex, en fonction du polissage éolien. L'abrasion éolienne sur des artéfacts en quartz a été expérimentée par Knutsson et Lindé [140] qui ont observé des fractures, des fissures et des stries caractéristiques. Macroscopiquement, la corrasion réduit les aspérités des éléments rocheux et peut façonner des facettes planes réunies par des angles émoussés (ventifacts). Dans les milieux à regs, les vestiges archéologiques peuvent alors se juxtaposer au sol aux autres fragments rocheux éolisés que sont les cailloux tétraédriques et dreikanters. Un autre effet du vent consiste au développement d'un vernis ou patine désertique (desert / rock varnish, [141]), produit généralement brillant, noir ou très foncé, de fine épaisseur et microstratifié, lié à l'accrétion de produits atmosphériques précipités sur les éléments rocheux, de composition organique et minérale (ferro-manganique). La conjugaison de ces différents facteurs peut effacer les caractéristiques technologiques et donc rendre difficile l'at- 
tribution culturelle des vestiges [137, 138]. Ugalde et al. [139], suivant une analyse macroscopique d'assemblages lithiques dans le désert d'Atacama (Chili), définissent sept stades de météorisation lithique en fonction du poli des arêtes et des bords (frais à émoussé), de la texture (degrés de rugosité) et de l'éclat (absent à intense). Les auteurs soulignent le rôle des micro-environnements (opposition interfluve / canyon) dans l'intensité et le type de météorisation.

\subsection{Impact des conditions périglaciaires}

La manifestation macroscopique la plus évidente des actions périglaciaires concerne la gélifraction qui a fait l'objet de nombreuses expérimentations tant sur les roches [142-146] que sur les vestiges fauniques [147-149]. Si la reconnaissance des altérations dues au gel sur de nombreuses séries lithiques paléolithiques apparait sans ambiguïté, les modalités de la gélifraction du silex restent cependant encore mal connues. De par sa faible porosité, le silex, y compris sous forme d'artéfacts et indépendamment du type de faciès, apparaît généralement peu gélif, excepté lorsqu'il est micro- ou macro-fissuré (ex. pour raisons tectoniques ou thermiques). Un gel brutal semble favoriser la formation de cupules alors qu'un gel progressif induirait une fragmentation plus efficiente du silex, d'autant plus marquée que les températures sont négatives. La fragmentation repose également sur le nombre de cycles gel-dégel, qui diminuent lorsque la température décroît, alors que l'efficacité du gel augmente. À l'échelle microscopique, certaines modifications des états de surface seraient en relation directe avec les processus cryogéniques [76, 146, 150, 151]. L'effet de la solifluxion sur les traces d'utilisation des outils lithiques a été testé expérimentalement à Gavarnie dans les Hautes-Pyrénées [152]. Dans le cadre de cette étude, qui porte sur des pièces en silex ayant évolué deux ans enterrées dans une coulée à front pierreux, aucune des altérations mises en évidence par des expériences en laboratoire [151, 153-155] n'a été observée. Ces données, qui rejoignent celles de Texier et al. [19], montrent que l'altération périglaciaire de la surface et des tranchants des objets peut être un mécanisme lent [146]. Par ailleurs, l'existence d'un poli de glace a été discutée par Caspar et al. [151] dans le cadre de l'analyse tracéologique de silex taillés non utilisés retrouvés dans des horizons de gley de toundra cryoturbés, sur le site de plein-air d'Hermies-Champs Bruquette (Pas-de-Calais). Le micropoli observé, réticulaire en dôme, et à surface lisse, est semblable à celui produit par le travail du bois, ce qui soulève le problème de la convergence morphologique des altérations taphonomiques et des traces fonctionnelles [156]. Divers expériences d'utilisation de silex taillés (raclage, sciage de glace ou de limon gelé) semblent en effet montrer le rôle de la glace ou d'un substrat minéral gelé dans la formation d'un micropoli [146, 151]. La cryoturbation ou la cryoexpulsion, en provoquant des mouvements typiques de la couche active des cryosols, pourraient expliquer cette modification de surface par les frottements de glace ou de substrat gelé qu'elles provoquent sur des objets lithiques [76, 151].

\subsection{Impact des écoulements liquides}

Les interactions artéfacts / dynamique fluviale ont souvent été évaluées à l'aide de différents attribus macroscopiques en caractérisant l'aspect de surface, par exemple la fraicheur du matériel (« roulé / frais »), l'abrasion, le degrés d'émoussé des bords, 
la largeur des arêtes ou encore la hauteur des crêtes d'enlèvement [73, 157-161]. Le degré d'abrasion des vestiges a classiquement été utilisé comme un indicateur chronologique et de distance de transport depuis le lieu d'abandon. Les bifaces possédant des arêtes fraîches, en association avec des éclats et des os de vertébrés ont souvent été interprétés comme des assemblages peu transportés, à la différence des bifaces aux arêtes émousées jugés en position secondaire et/ou plus âgés [162-166]. Pour certains auteurs, cependant, la dichotomie entre position primaire et secondaire n'est pas toujours valide $[17,72,167]$, alors que la relation entre le contexte dépositionnel et l'impact des écoulements sur les vestiges apparaît complexe. Le transport fluviatile est par nature épisodique ou intermittent, incluant potentiellement de multiples phases d'enfouissement et de remaniement de vestiges (ex. gisements acheuléens à bifaces). L'interprétation des assemblages, et en particulier l'évaluation de leurs intégrités spatiale et chronologique, est donc dépendante des conditions de préservation des artéfacts, mais aussi de la nature des paléoenvironnements fluviatiles et des conditions dépositionnelles locales [168, 169].

La reconnaissance des dommages induit par le transport fluviatile a fait l'objet de plusieurs expériences en laboratoire (ex. canaux, tambours) mais aussi en milieu naturel [157-159, 161, 169-173]. Shackley [157] propose sept stades d'abrasion fondés sur l'observation microscopique des arêtes de pièces en silex, en insistant sur quatre facteurs : la dureté du silex, la vitesse d'écoulement, la forme de l'artéfact, et la texture et la lithologie du lit du chenal. Deux mécanismes d'attaque mécanique en découlent : la fracturation par effet de collision entre clastes, et la réduction progressive des arêtes émergentes par le pouvoir abrasif des particules fines en mouvement (limon, sable). Harding et al. [158] ont réalisé une expérience en disposant pendant plusieurs mois des répliques de bifaces dans le lit d'un cours d'eau à charge de fond constituée de galets. L'intensité et la distribution des altérations (encoches, écaillures, polis, points de percussion) sont corrélés avec la distance de transport et le poids du silex des bifaces. La perte de poids des objets augmente avec la distance de transport parcourue en lien avec le détachement d'éclats par les chocs répétés avec des clastes naturels grossiers. L'acquisition d'une forme ronde, par enlèvements répétés, permettrait à un émoussé de se développer. L'influence de la forme des objets sur le transport est plus difficile à caractériser, les objets de forme ovale seraient davantage enclins à un transport lointain que ne le sont les pièces allongées. Par ailleurs, la distance parcourue par les bifaces semble déterminée par leur localisation de départ dans le système fluvial, en fonction de la vitesse d'écoulement et de la topographie du lit. Le poli de surface, initialement observé sur les arêtes et facettes plates, semble se former rapidement, sur une courte distance de transport, pour ensuite gagner en intensité et distribution.

Pour les sites paléolithiques, une des limitations de l'approche expérimentale concerne la difficulté à reproduire les conditions d'écoulement de haute énergie typiques des rivières périglaciaires et par conséquent, à obtenir des états de surface comparables à ceux d'artéfacts retrouvés dans certains contextes archéologiques [174, 175]. En outre, il semble difficile de convertir des heures d'expériences en canal en distance de transport en rivière. Les impacts macro- et microscopiques des écoulements de faible énergie sur les vestiges sont également peu connus [170], paramètre déterminant lorsque l'on juge l'état de fraîcheur du matériel et son caractère in situ. Sur ce point, les expé- 
riences en tambours $[157,161]$ ne pouvant pas directement reproduire les différents modes de transport, sont davantage repésentatives d'écoulement de très haute énergie. Si les études expérimentales montrent que le transport fluviatile peut opérer sans engendrer des signes d'abrasion macroscopiques [157, 176, 177], les écoulements de faible énergie sont néanmoins susceptibles d'induire des microtraces d'abrasion [170]. Par ailleurs, à l'inverse de ce qui a parfois été suggéré [157], les artefacts n'ont pas besoin d'être mobiles pour montrer des traces d'usure ou des bords endommagés. Selon Chu et al. [170], les microtraces d'abrasion sur le silex (micro-écaillage, attaque des arêtes) mais aussi les marques de percussion ou de fracturation seraient davantage reliées à l'énergie de l'écoulement et au temps passé dans le milieu fluviale qu'au transport des artéfacts et à la distance parcourue. Le flux de particules passant sur un artéfact immobilisé peut donc suffir à modifier sa surface comme c'est le cas des vestiges piégés en fond de chenal [167, 177]. En considérant la granulométrie du matériau transportée par l'écoulement, Petraglia et Potts [167] proposent un modèle de développement de l'abrasion des vestiges selon trois scénarios. Le premier concerne le cas d'artéfacts mobiles qui rentrent en contact avec des particules sédimentaires fines durant le transport, provoquant une attaque des surfaces, des bords et des arêtes, avec un émoussé [82]. Dans le second, lorsque les objets sont immobiles sur le lit de la rivière ou partiellement enfouis, les surfaces exposées aux particules fines mobiles sont émoussées alors que les arêtes protégées peuvent rester fraîches (ex. sites en contexte de berges sableuses [178]). Dans le troisième scénario, lorsque les artéfacts sont transportés en charge de fond avec des éléments grossiers (cailloux, galets, blocs) avec lesquels ils s'entrechoquent, les bords se fracturent [158] ou s'émoussent.

Enfin, concernant l'impact du ruissellement sur l'état de surface des objets lithiques, les expériences de Lenoble [23] en tambour et en plein champ montrent qu'une altération par abrasion de l'état de surface des silex taillés est possible. Cette altération, surtout liée au ruissellement concentré, est provoquée par les chocs répétés du sédiment transporté avec les vestiges qui forment une fraction résiduelle plus ou moins mobile. Elle à l'origine d'un lustre des vestiges archéologiques et d'un émoussé de leurs parties saillantes.

\subsection{Altération chimique}

L'altération chimique post-dépositionnelle peut modifier, détruire, voire imiter les signatures fonctionnelles des vestiges lithiques, rendant l'étude des micro-traces d'utilisation difficile ou impossible. À titre d'exemple, l'altération de silex taillés paléolithiques peut favoriser le développement d'une forte porosité observable au MEB, avec des pores de très grande taille, donnant une couleur blanche au silex [179]. En Inde, la formation de croûtes ferrugineuses (ferricretes) tend à détériorer les artéfacts paléolithiques en quartzite ou en grès quartzeux, avec le développement d'un cortex d'altération mais aussi d'une patine, notamment dans le grès [180]. À la surface des vestiges en silex, les modifications visuelles, morphologiques et minéralogiques, et plus particulièrement la formation de patines, sont des mécanismes complexes (dissolution différentielle et reprécipitation de la silice, réorganisation des phases siliceuses, hydratation, oxydation) dépendant de plusieurs facteurs pétrographiques, stationnels, bio-pédologiques mais aussi anthropiques [129-133, 153, 181-185]. Citons le potentiel 
de mise en solution de la silice du milieu, notamment le rôle du degrés d'alcalinité $(\mathrm{pH}) \mathrm{du}$ microenvironnement, avec les effets de la chimie de l'eau de pluie, du sol et du sous-sol, mais aussi : la vitesse d'enfouissement des vestiges, le temps d'exposition aux attaques photochimiques (rôle des UV dans la dissolution du quartz cryptocristallin), l'activité bactérienne, le type de substances et d'acides organiques (ex. substances humiques, exsudats des racines végétales : citrate, oxalate, acétate), la présence d'acides aminés basiques (histidine, arginine, lysine), l'histoire thermique du sol (ex. la solubilité du quartz décroit de façon exponentielle avec la température), ou encore la qualité du drainage et la position topographique (influence de la catena et de l'exposition des versants). À cela s'ajoutent comme facteurs de contrôle, l'influence de la microstructure du matériau siliceux (porosité, ciment interstitiel, texture, taille des lépisphères), sa composition minéralogique (types d'impuretés), le degré de cristallinité et les formes cristallines de la silice (ex. silice amorphe / opale A, opale CT, calcédoine, moganite, quartz automorphe : rôle déterminant dans la solubilité et la stabilité des phases), la percolation d'eau à travers le matériau, le temps écoulé de la diagenèse, ou encore l'existence d'une chauffe de l'artéfact (traitement thermique anthropique ou feu naturel).

La complexité taphonomique réside dans le fait que dans certains cas, les attaques chimiques sont susceptibles de rendre les vestiges plus sensibles aux altérations mécaniques. Inversement, le développement de microfractures, de stries, ou de trous d'origine mécanique (naturelle ou anthropique; ex : rainures de broyage ou polissage) peut favoriser l'attaque chimique et l'apparition d'une patine, en favorisant la pénétration de l'eau. Alors que les vitesses de dissolution et de précipitation de la silice sont généralement lentes (notamment dans les sols tempérés), la surface des artéfacts en silex peut rapidement montrer une altération microcristalline, d'ordre inframillimétrique, suite à des phénomènes de percolation d'eaux ultra-basiques $(\mathrm{pH}>9 ;[186])$ ou même théoriquement peu agressives vis à vis de la silice $[179,187]$. Les conditions alcalines sont communes dans les sols riches en coquilles, dans les dépôts à cendres volcaniques (tephras) ou d'origine anthropique (foyers), notamment lorsque du bois, de la tourbe ou des excréments d'herbivores (ex. vaches) ont été utilisés comme combustibles. D'une manière générale, les solutions alcalines, d'origine naturelle ou anthropique (ex. solutions ammoniaquées : macération, urine) fragilisent les polis et participent à la conservation différentielle des traces d'utilisation en fonction de la roche, de la matière travaillée (os, bois, peau, viande), et de la forme du tranchant [153-155, 182, 188-190]. Un $\mathrm{pH}$ très acide ou basique n'est cependant pas toujours nécessaire pour provoquer la dissolution de la silice [155], notamment en cas de présence d'impuretés solubles à des $\mathrm{pH}$ neutres. De plus, il existe une solubilité différentielle des différentes formes de silice, qui sont elles-mêmes moins solubles que la silice amorphe [185]. Précisons aussi que l'altération des silex conduit à une perte en silice, même si en retour la cristallinité et la stabilité des minéraux siliceux augmentent [stabilité des phases : 185]. Si la dissolution de la silice s'observe généralement à la surface des silex enfouis, sa précipitation peut avoir lieu suite à un assèchement du sol ou au refroidissement d'une nappe d'eau remontante (comme sous climat périglaciaire).

La présence d'eau, particulièrement dans des conditions d'hydromorphie marquée, favorise le développement d'une patine blanche (white patina), liée à la désilicification, 
se caractérisant par le développement d'un voile blanchâtre recouvrant la totalité du vestige altéré. Ce type de patine correspond microscopiquement à une dispersion importante de la lumière, à une absence de contraste, et à la disparition de la microstructure. La dissolution peut induire la précipitation de composés insolubles à la surface du silex, incluant la silice sous forme de laminations, lorsque la solution est saturée de silice à proximité de la surface. On peut noter aussi le développement d'une patine brillante (gloss patina), ou lustre, lorsque le vestige montre une brillance micro- et/ou macroscopique qui est surtout présente sur les points saillants de la pièce (arête, tranchant, bulbe). Ce type de patine, qui tend à réduire ou adoucir la topographie de la surface des artéfacts, est également généré par une dissolution de la silice dont les effets sont proches de l'altération rencontrée en rivière par abrasion [130, 131, 181]. Si l'adsorption de silice amorphe par les vestiges, à partir du microenvironnement, peut produire une patine brillante, la dissolution peut aussi être concomitante de processus de re-précipitation à la surface des artéfacts [131]. Des spots brillants (bright spots) vont désigner des plages très brillantes de polis à coalescence dure et généralement plate qui sont aléatoirement distribuées sur la surface ou les bords des vestiges. Ces spots brillants sont parfois interprétés comme le résultat de la déposition et de la solidification d'un gel de silice colloïdale du sol ayant précipité in situ dans le microenvironnement des vestiges [130].

Pour certains auteurs, la patine brillante, les spots brillants et la patine blanche seraient les effets successifs de la dissolution et du dépôt de la silice, mécanisme pouvant être observé en présence de solutions de carbonate de calcium et de sodium $[153,154]$. Une patine dendritique (vermiculé, curvilinéaire) est plutôt interprétée comme le résultat de l'action combinée des acides organiques, des acides aminés, et des flux de carbonates, dans la rhizosphère (ex. des vestiges paléolithiques en contexte lœessique : [133]; voir aussi [153] sur le rôle des racines). Par ailleurs, l'apparition de la patine, pendant la dissolution d'un verre siliceux, serait en lien avec la formation de couches hydratées. Durant ce processus, les impuretés (oxydes de manganèse, fer, sulfure de fer, minéraux argileux, carbonates) seraient dispersées puis illuviées dans le silex suivant des profondeurs et des densités variables [133].

Enfin, les études menées en laboratoire tendent à montrer que ni le type de patine, ni l'épaisseur de celle-ci, ne peuvent être utilisés pour dater le matériel lithique, ce que confirment les données de terrain [129, 132, 181, 191-194].

\section{Conclusion et perspectives}

L'approche taphonomique du matériel archéologique reste encore peu développée; des progrès notables peuvent donc être attendus au fur et à mesure de l'avancement des recherches dans cette voie. Certains types d'analyse, comme la distribution des distances de raccords et leur orientation, devraient pouvoir apporter des informations intéressantes afin de compléter le panel d'outils disponibles pour mieux comprendre les processus de formation des sites. Néanmoins, l'un des points les plus cruciaux pour progresser est d'obtenir de nouveaux référentiels expérimentaux sur les types de transformations occasionnées par les processus tant sédimentaires que biologiques. Pour être réellement exploitables, ces expériences doivent utiliser des répliques de ma- 
tériel archéologique et doivent permettre de mesurer les perturbations subies par des assemblages de vestiges présentant une configuration initiale proche de celles connues dans les sites archéologiques. En effet, l'une des difficultés pour utiliser en archéologie les données issues de la géomorphologie vient du fait que les dépôts naturels correspondent la plupart du temps à des accumulations de matériaux qui ont enregistré un transport pendant une longue période et qui ont subi plusieurs phases de remobilisation avant d'être enfouis. Une occupation humaine se traduit en revanche par des apports de nouveaux matériaux dans un contexte sédimentaire où des organisations spécifiques (tri granulométrique, fabrique... ) peuvent déjà exister. Cette occupation ne sera reconnue comme site archéologique que dans la mesure où la redistribution des objets a été faible, en d'autres termes, si le site n'a été exposé aux écoulements que pendant une durée brève et qu'il a été rapidement enfoui. Le recours à des expériences semble donc la meilleure approche pour caractériser les premiers stades de redistribution et obtenir des données utilisables en archéologie. Un autre point important est la morphologie généralement très aplatie des objets archéologiques (ex. éclats, lames, fragments de diaphyses d'os longs). Cette morphologie influe sensiblement sur leur comportement vis à vis de différents processus (cryoexpulsion, écoulements...), qui ne peut donc être directement comparé à celui des graviers naturels de forme généralement arrondie. En l'état actuel, les données expérimentales dédiées à l'archéologie sont assez réduites. Ce champ d'investigation est vaste et l'on est encore loin de disposer d'une vue d'ensemble des types de perturbation possibles.

Deux points importants concernant l'intérêt pour le futur des études taphonomiques sur les sites paléolithiques doivent être soulignés :

- Une étude critique des conditions de conservation des sites qui ont donné lieu à une analyse spatiale poussée des vestiges, visant à reconstituer la configuration de l'habitat, doit être entreprise. Il est probable que nombre de ces sites s'avèrent, après analyse taphonomique, ne pas constituer des sources fiables de documentation, parce que l'impact des processus naturels dans la formation du niveau archéologique a été sous-estimé par le passé. Ce point est d'autant plus crucial que les périodes concernées sont anciennes et sont associées à des hominidés dont les capacités cognitives restent largement inconnues. C'est en particulier le cas en France pour les Néandertaliens.

- Des analyses techno-économiques et archéozoologiques très détaillées sont réalisées actuellement sur les sites paléolithiques, de manière à comprendre la gestion des matières premières lithiques ou fauniques à l'échelle du site mais aussi à l'échelle du territoire (importation - exportation de produits à des stades variés d'élaboration). Ces analyses ne prennent tout leur sens que si une analyse taphonomique permet de démontrer l'intégrité de l'assemblage et l'absence de tri par les processus naturels. Les développements récents de l'analyse granulométrique du matériel lithique ou osseux indiquent sans ambiguïté que cela est loin d'être le cas général. 


\section{Références}

[1] W.R. Wood, D.L. Johnson, A survey of disturbance processes in archaeological site formation. Advances in Archaeological Methods and Theory, 1, 315-381, 1978.

[2] K. Butzer, Archaeology as human ecology. Cambridge University Press, Cambridge, 364 p., 1982.

[3] M.B. Schiffer, Toward the identification of site formation processes. American Antiquity, 48, 675-706, 1983.

[4] M.B. Schiffer, Formation processes of the archaeological record. University of New Mexico Press, Albuberque, 427 p., 1987.

[5] M.R. Waters, Principles of geoarchaeology. The University of Arizona Press, Tucson, 399 p., 1992.

[6] P. Bertran, J.P. Texier, « Dépôts de pente et archéologie », dans Dynamique du paysage, Entretiens de géoarchéologie, J.P. Bravard et M. Prestreau (eds.), DARA, Lyon, 59- 86, 1997.

[7] J.P. Texier, À propos des processus de formation des sites préhistoriques. Paleo, 1, 379-386, 2000.

[8] J.P. Texier, Histoire géologique des sites préhistoriques classiques du Périgord : une vision actualisée. La Micoque, la grotte Vauffrey, le Pech de l'Azé I et II, La Ferrassie, l'abri Castenet, Le Flageolet, Laugerie Haute. Documents préhistoriques 25, CTHS, 193 p., 2009.

[9] J.G. Bordes, « Lithic taphonomy of the Châtelperronian / Aurignacian interstratifications in Roc de Combe and Le Piage (Lot, France) », dans The chronology of the Aurignacian and transitional technocomplexes. Dating, stratigraphies, cultural implications, Trabalhos de Arqueologia 33, Instituto Português de Arqueologia, Lisboa, 223-244, 2003.

[10] P. Villa, Taphonomy and stratigraphy in European prehistory. Before Farming, 1, 1-19, 2004.

[11] I.A. Efremov, Taphonomy : a new branch of paleontology. Pan American Geologist, 74, 81-93, 1940.

[12] R.G. Whitlam, « Archaeological taphonomy : implications for defining data requirements and analytical procedures » dans Directions in Archaeology : A Question of Goals, P.D. Francis, E.C. Poplin (eds.), Calgary, Alberta, 145- 154, 1982.

[13] R.L. Lyman, What taphonmy is, what it isn't, and why taphonomists should care about the difference. Journal of Taphonomy 8 (1), 1-16, 2010

[14] M. Dominguez-Rodrigo, S. Fernandez-Lopez, L. Alcala, How can Taphonomy be defined in the XXI century? Journal of Taphonomy 9 (1), 1-13, 2011.

[15] H. Dibble, P. Chase, S. McPherron, A. Tuffreau, Testing the reality of a "living floor" with archaeological data. American Antiquity, 62, 629-651, 1997.

[16] Ph. Crombé, J. Sergant, J. De Reu, La contribution des dates radiocarbone pour démêler les palimpsestes mésolithiques : exemples provenant de la région des sables de couverture en Belgique du Nord-Ouest. Bulletin de la Société Préhistorique Française, 1-2, 235-250, 2013.

[17] K.D. Schick, Stone Age sites in the making. Experiments in the formation and transformation of archaeological occurrences. BAR International Series 319, Oxford, 313 p., 1986.

[18] P. Bowers, R. Bonnichsen, D. Hoch, Flake dispersal experiments : noncultural transformation of the archaeological record. American Antiquity 48, 553-572, 1983.

[19] J.P. Texier, P. Bertran, J.P. Coutard, B. Francou, P. Gabert, J.L. Guadelli, J.C. Ozouf, H. Plisson, J.P. Raynal, D. Vivent, TRANSIT, an experimental archaeological program in periglacial environment : problematic, methodology, first results. Geoarchaeology : An International Journal, 13, 433-473, 1998.

[20] M.R. Hilton, Quantifying postdepositional redistribution of the archaeological record produced by freeze-thaw and other mechanisms : an experimental approach. Journal of Archaeological Method and Theory, 10 (3), 165-202, 2003.

[21] A. Lenoble, P. Bertran, F. Lacrampe, Solifluction-induced modifications of archaeological levels : simulation based on experimental data from a modern periglacial slope and application to French Palaeolithic sites. Journal of Archaeological Science, 35, 99-110, 2008.

[22] P. Bertran, C. Beauval, S. Boulogne, M. Brenet, S. Costamagno, T. Feuillet, V. Laroulandie, A. Lenoble, Ph. Malaurent, J.B. Mallye, Experimental archaeology in a mid-latitude periglacial context : insight into site formation and taphonomic processes. Journal of Archaeological Science, 57, 283-301, 2015.

[23] A. Lenoble, Ruissellement et formation des sites préhistoriques : référentiel actualiste et exemples d'application au fossile. BAR International Series 1363, Oxford, 212 p., 2005. 
[24] D. Cahen, Das zusammensetzen geschlagener Steinartefakte. Archäologisches Korrespondenzblatt, 6, 81-93, 1976.

[25] H.T. Bunn, J.W.K. Harris, G. Isaac, Z. Kaufulu, E. Kroll, K. Schick, N. Toth, A.K. Behrensmeyer, FxJj50 : An early Pleistocene site in Northern Kenya. World Archaeology, 12, 109-136, 1980.

[26] R.N.E. Barton, « Vertical distribution of artefacts and some post-depositional factors affecting site formation », dans Mesolithic Northwest Europe : recent trend, P. Rowley-Conwy, M. Zvelebil, H.P. Blankholm (eds.), University of Sheffield, Department of Archaeology and Prehistory, 55-62, 1987.

[27] P. Villa, Conjoinable pieces and site formation processes. American Antiquity, 47 (2), 276-290, 1982.

[28] F. Van Noten, D. Cahen, L. Keeley, A Paleolithic campsite in Belgium. Scientific American, 242, 44-51, 1980.

[29] J.L. Hofman, Vertical movement of artifacts in alluvial and stratified deposits. Current Anthropology, $27(2), 163-171,1986$.

[30] P.M. Vermeersch, S. Bubel, Postdepositional artefact scattering in a podzol : processes and consequences for Late Palaeolithic and Mesolithic Sites. Anthropologie, XXXV (2/3), 119-130, 1997.

[31] T.A. Surovell, N.M. Waguenspack, J.H. Mayer, M. Kornfeld, G.C. Frison, Shallow site archaeology : artifact dispersal, stratigraphy, and radiocarbon dating at the Barger Gulch Locality B Folsom site, Middle Park, Colorado. Geoarchaeology : An International Journal, 20, 627-649, 2005.

[32] F. Bordes, Comptes Rendus critique de H. de Lumley de « La Grotte de l'Hortus ». Quaternaria, XVI, 299-305, 1972 .

[33] M.D. Petraglia, K. Akoshima, L.G. Strauss, Interpreting the formation of the Abri Dufaure : an Upper Paleolithic site in Southwestern France. Journal of Anthropological Archaeology, 13, 139-151, 1994.

[34] F.P. Hole, Effects of animals on soils. Geoderma, 25, 75-112, 1981.

[35] B. Bocek, The Jasper Ridge reexcavation experiment : rates of artifact mixing by rodents. American Antiquity, 57 (2), 261-269, 1992.

[36] M. Armour-Chelu, P. Andrews, Some effects of bioturbation by earthworms (Oligocheta) on archaeological sites. Journal of Archaeological Science, 21, 433-443, 1994.

[37] C.L. Balek, Buried artifacts in stable upland sites and the role of bioturbation : a review. Geoarchaeology : An International Journal, 17 (1), 41-51, 2002.

[38] D.L. Johnson, Darwin would be proud : bioturbation, dynamic denudation, and the power of theory in science. Geoarchaeology : An International Journal, 17 (1), 7-40, 2002.

[39] A.G. Araujo, J.C. Marcelino, The role of armadillos in the movement of archaeological materials : an experimental approach. Geoarchaeology : An International Journal, 18 (4), 433-460, 2003

[40] M.G. Canti M.G., Earthworm activity and archaeological stratigraphy : a review of products and processes. Journal of Archaeological Science, 30, 135-148, 2003.

[41] J.B. Mallye, «Les restes de Blaireau en contexte archéologique : taphonomie, archéozoologie et éléments de discussion des séquences préhistoriques », Thèse de doctorat, Université Bordeaux 1, Talence, 547 p., 2007.

[42] P. Villa, J. Courtin, The interpretation of stratified sites : a view from the underground. Journal of Archaeological Science, 10, 267-281, 1983.

[43] A.E. Nielsen, Trampling the archaeological record : an experimental study. American Antiquity, 56, 483-503, 1991.

[44] C. Thiébaut, S. Costamagno, M.P. Coumont, V. Mourre, N. Provenzano, I. Thery-Parisot, Approche expérimentale des conséquences du piétinement des grands herbivores sur les vestiges lithiques et osseux. Paléo, Supplément 3, 109-129, 2010.

[45] L. Asryan, A. Ollé, N. Moloney, Reality and confusion in the recognition of post-depositional alterations and use-wear : an experimental approach on basalt tools. Journal of Lithic Studies, 1 (1), 9-32, 2014.

[46] R.J. Schaetzl, S.F. Burns, T.W. Small, D.L. Johnson, Tree uprooting : review of types and patterns of soil disturbance. Physical Geography, 11, 277-291, 1990.

[47] P. Crombé, Tree-fall features on final Palaeolithic and Mesolithic sites situated on sandy soils : how to deal with it. Helinium, 33, 50-66, 1993. 
[48] R. Langohr, Types of tree windthrow, their impact on the environment and their importance for the understanding of archaeological excavation data. Helinium, XXXIII/1, 36-49, 1993.

[49] J.D. Phillips, D.A. Marion, A.V. Turkington, Pedologic and geomorphic impacts of a tornado blowdown event in a mixed pine-hardwood forest. Catena, 75, 278-287, 2008.

[50] A. Delagnes, A. Lenoble, S Armand, J.P. Brugal, S. Prat, J.J. Tiercelin, H. Roche, Interpreting pachyderm single carcass sites in the African Lower and Early Middle Pleistocene record : a multidisciplinary approach to the site of Nadung'a 4 (Kenya). Journal of Anthropological Archaeology, 25 (4), 448-465, 2006.

[51] D. Cahen, J. Moeyersons, Subsurface movements of stone artefacts and their implications for the prehistory of Central Africa. Nature, 266 (5605), 812-815, 1977.

[52] D.L. Johnson, P.V. Hansen, The effects of frost-heaving on objects in soils. Plains Anthropologist, 19, $81-98,1974$

[53] A. Pissart, Apparition et évolution des sols structuraux périglaciaires de haute-montagne. Expériences de terrain au Chambeyron (Alpes, France). Abhandlungen der Akademie der Wissenschaften zu Göttingen, Mth.-Phys., Kl., 3F (31), 142-156, 1977.

[54] B. Masson, Structures de combustion et structures périglaciaires : ré-examen taphonomique des structures de combustion moustériennes de Saint-Vaast-La-Hougue (50).P@lethnologie, 2, 5-23, 2010.

[55] C. Yamagishi, N. Matsuoka, Laboratory frost sorting by needle ice : a pilot experiment on the effects of stone size sorting and extent of surface stone cover. Earth Surface processes and Landforms, 40, 502-511, 2015 .

[56] D. Bideau, « Introduction générale aux milieux granulaires », dans Des grands écoulements naturels à la dynamique du tas de sable. Introduction aux suspensions en géologie et en physique. B. Ildefonse, C. Allain et P. Coussot (eds.), CEMAGREF, Antony, 193-204, 1997.

[57] K.D. Fowler, H.J. Greenfield, L.O. van Schalkwyk, The effects of burrowing activity on archaeological sites : Ndondondwane, South Africa. Geoarchaeology, 19, 441-470, 2004.

[58] L. Bueno, J. Feathers, P. De Blasis, The formation process of a paleoindian open-air site in Central Brazil : integrating lithic analysis, radiocarbon and luminescence dating. Journal of Archaeological Science, 40, 190-203, 2013

[59] E. Morin, Beyond stratigraphic noise : unravelling the evolution of stratified assemblages in faunalturbated sites. Geoarchaeology : An International Journal, 21 (6), 541-565, 2006.

[60] D.L. Johnson, Subsurface stone lines, stone zones, artifact-manuport layers, and biomantles produced by bioturbation via Pocket Gophers (Thonomys bottae). American Antiquity, 54, 370-389, 1989.

[61] D.L. Johnson, Biomantle evolution and the redistribution of earth materials and artifacts. Soil Science, 149, 84-102, 1990

[62] M.A.J. Williams, «Termites, soils and landscape equilibrium in the Northern Territory of Australia » dans Landform Evolution in Australasia, J.L. Davies, M.A.J. Williams (eds.), Canberra, Australian National University Press, 128-141, 1978.

[63] D. Schwartz, Archéologie préhistorique et processus de formation des stone-lines en Afrique centrale (Congo-Brazzaville et zones périphériques). Géo-Eco-Trop, 20 (1-4), 15-38, 1996.

[64] J. Mercader, R. Marti, J. Martinez, A. Brooks A., The nature of stone-lines in the African Quaternary record : archaeological resolution at the rainforest site of Mosumu, Equatorial Guinea. Quaternary International, 89, 71-96, 2001.

[65] S.N. Collcutt, N.R.E. Barton, C.A. Bergman, « Refitting in context : a taphonomic case study from a Late Upper Palaeolithic site in sands on Hengistbury Head, Dorset, Great Britain » dans The Big Puzzle. International Symposium on Refitting Stone Artefacts, E. Cziesla, S. Eickhoff, N. Arts et D. Winter (eds.), Holos, Bonn, 219-235, 1990.

[66] L. Sitzia, P. Bertran, S. Boulogne, M. Brenet, R. Crassard, A. Delagnes, M. Frouin, C. Hatté, J. Jaubert, L. Khalidi, E. Messager, N. Mercier, A. Meunier, S. Peigné, A. Queffelec, C. Tribolo, R. Macchiarelli, The paleoenvironment and lithic taphonomy of Shi'Bat Dihya 1, a Middle Paleolithic site in Wadi Surdud, Yemen. Geoarchaeology : An International Journal, 27, 471-491, 2012.

[67] P. Antoine, J. Catt, J.P. Lautridou, J. Sommé, The loess and coversands of northern France and southern England. Journal of Quaternary Science, 18 (3), 309-318, 2003.

[68] P. Haesaerts, F. Damblon, N. Gerasimenko, P. Spagna, S. Pirson, The Late Pleistocene loess-palaeosol sequence of Middle Belgium. Quaternary International, 1-19, 2016. 
[69] A. Leroi-Gourhan, Dictionnaire de la Préhistoire. Paris, PUF, 1222 p., 1988.

[70] N. Arts, E. Cziesla, « Bibliography (1880-1988) on the subject of refitting stone artefacts » dans The Big Puzzle. International Symposium on Refitting Stone Artefacts, E. Cziesla, S. Eickhoff, N. Arts, D. Winter (eds.), Holos, Bonn, 651-683, 1990.

[71] M. Frouin, C. Lahaye, M. Hernandez, N. Mercier, P. Guibert, M. Brenet, M. Folgado-Lopez, P. Bertran, Chronology of the Middle Palaeolithic open-air site of Combe Brune 2 (Dordogne, France) : a multi luminescence dating approach. Journal of Archaeological Science, 52, 524-534, 2014.

[72] K.D. Schick, « Experimentally-derived criteria for assessing hydrologic disturbance of archaeological sites » dans Natural formation processes and the archaeological record, D.T. Nash et M.D. Petraglia (eds.), BAR International Series 352, Oxford, 86-107, 1987.

[73] J.J. Shea, Artifact abrasion, fluvial processes, and "living floors" from the early Paleolithic site of 'Ubeidiya (Jordan Valley, Israel). Geoarchaeology : An International Journal, 14 (2), 191-207, 1999.

[74] G. Boschian, D., Saccà, Ambiguities in human and elephant interactions? Stories of bones, sand and water from Castel di Guido (Italy). Quaternary International, 214, 3-16, 2010.

[75] K.A. Adelsberger, J.R. Smith, S.P. McPherron, H.L. Dibble, D.I. Olszewski, U.A. Schurmans, L. Chiotti, Desert pavement disturbance and artifact taphonomy : a case study from the Eastern Libyan Plateau, Egypt. Geoarchaeology : An International Journal, 28 (2), 112-130, 2013.

[76] J.P. Caspar, B. Masson, L. Vallin, Taphonomie des ensembles lithiques du Paléolithique moyen en contexte loessique. L'approche expérimentale. Les Nouvelles de l'Archéologie, 118, 21-26, 2009.

[77] P. Bertran, L. Klaric, A. Lenoble, B. Masson, L. Vallin, The impact of periglacial processes on Palaeolithic sites : the case of sorted patterned grounds. Quaternary International, 214, 17-29, 2010.

[78] B. Masson, L. Vallin, Altération des sols paléolithiques par la formation de sols figurés sous climat périglaciaire : approches expérimentale et illustrations archéologiques. Paléo, Supplément 3, 77-92, 2010.

[79] B. Masson, L. Vallin, Ph. Feray, «Impacts des phénomènes périglaciaires sur la conservation des sites archéologiques »dans Manuel de Taphonomie, C. Denys et M. Patou-Mathis (eds.), Errance, Collection Archéologiques, 227-250, 2014.

[80] J. Sommé, J. Vaillant, J.P. Fagnart Contribution à l'étude du gisement moustérien de Solesmes (Nord). Bulletin de la Société Préhistorique Française, 69 (2), 481-491, 1972.

[81] P. Bertran, A. Lenoble, F. Lacrampe, M. Brenet, C. Cretin, F. Milor, Le site aurignacien de Combemenue à Brignac-la-Plaine (Corrèze) : apport de la géoarchéologie et de l'étude de l'industrie lithique à la compréhension des processus taphonomiques. Paléo, 17, 7-29, 2005.

[82] G.L. Isaac Towards the interpretation of occupation debris : some experiments and observations. The Kroeber Anthropological Society Papers, 37, 31-57, 1967.

[83] O. Bar Yosef, E. Tchernov, « On the palaeo-ecological history of the site of Ubeidiya, Israel ». Publications of the Israel Academy of Sciences and Humanities, Jerusalem, Israël, 35 p., 1972.

[84] Z. Kaufulu, Formation and preservation of some earlier Stone Age sites at Koobi Fora, northern Kenya. The South African Archaeological Bulletin, 42, 23-33, 1987.

[85] P. Bertran, Dégradation des niveaux d'occupation paléolithiques en contexte périglaciaire : exemples et implications archéologiques. Paléo, 6, 285-302, 1994.

[86] P. Bertran, J.P. Texier, J.P., Fabric analysis : application to palaeolithic sites. Journal of Archaeological Science, 22, 521-535, 1995.

[87] P. Bertran, B. Hétu, J.P. Texier, H. Van Steijn, Fabric characteristics of slope deposits. Sedimentology, $44,1-16,1997$.

[88] A. Lenoble, P. Bertran, Fabric of Palaeolithic levels : methods and implications for site formation processes. Journal of Archaeological Science, 31, 457-469, 2004.

[89] S.J.P. McPherron, Artifact orientations and site formation processes from total station proveniences. Journal of Archaeological Science, 32, 1003-1014, 2005.

[90] P. Bertran, J.G. Bordes, A. Barré, A. Lenoble, V. Mourre, Fabrique d'amas de débitage : données expérimentales. Bulletin de la Société Préhistorique Française, 103, 33-47, 2006.

[91] A. Benito-Calvo, I. de la Torre Analysis of orientation patterns in Olduvai Bed I assemblages using GIS techniques : implications for site formation processes. Journal of Human Evolution, 61, 50-60, 2011. 
[92] M. Dominguez-Rodrigo, H.T. Bunn, T.R. Pickering, A.Z.P. Mabulla, C.M. Musiba, E. Baquedano, G.M. Ashley, F. Diez-Martin, M. Santonja, D. Uribelarrea, R. Barba, J. Yravedra, D. Barboni, C. Arriaza, A. Gidna, Autochthony and orientation patterns in Olduvai Bed I : A reexamination of the status of postdepositional biasing of archaeological assemblages from FLK north (FLKN). Journal of Archaeological Science, 39, 2116-2127, 2012.

[93] M. Dominguez-Rodrigo, D. Uribelarrea, M. Santonja, H.T. Bunn, A. Garcia-Pérez, A. Pérez-Gonzalez, J. Panera, S. Rubio-Jara, A. Mabulla, E. Baquedano, J. Yravedra, F. Diez-Martin, Autochtonous anisotropy of archaeological materials by the action of water : experimental and archaeological reassessment of the orientation patterns at the Olduvai sites. Journal of Archaeological Science, 41, 44-68, 2014.

[94] I. De la Torre, A. Benito-Calvo, Application of GIS methods to retrieve orientation patterns from imagery; a case study from Beds I and II, Olduvai Gorge (Tanzania). Journal of Archaeological Science, 40, 2446-2457, 2013.

[95] E. Batschelet, Circular statistics in biology. Academic Press, London, 371 p., 1981.

[96] N.I. Fisher, Statistical analysis of circular data. Cambridge University Press, Cambridge, 277 p., 1993.

[97] J.R. Curray, The analysis of two-dimensional orientation data. Journal of Geology, 64, 117-131, 1956.

[98] N.H. Woodcock, Specification of fabric shapes using an eigenvalue method. Geological Society of America Bulletin, 88, 12311236, 1977.

[99] D.I. Benn, D.I., Fabric shape and the interpretation of sedimentary data. Journal of Sedimentary Research A64, 4, 910-915, 1994.

[100] P. Bertran, E. Claud, L. Detrain, A. Lenoble, B. Masson, L. Vallin, Composition granulométrique des assemblages lithiques. Application à l'étude taphonomique des sites paléolithiques. Paléo, 18, 7-36, 2006.

[101] P. Bertran, A. Lenoble, D. Todisco, P.M. Desrosiers, M. Sørensen, Particle size distribution of lithic assemblages and taphonomy of Palaeolithic sites. Journal of Archaeological Science, 39, 3148-3166, 2012.

[102] P.V. Hansen, B.O. Madsen, Flint axe manufacture in the Neolithic. An experimental investigation of a flint axe manufacture at Hastrup Vaenget, East Zealand. Journal of Danish Archaeology, 2, 43-59, 1983.

[103] D.W. Stahle, J.E. Dunn, An analysis and application of the size distribution of waste flakes from the manufacture of bifacial stone tools. World Archaeology, 14 (1), 84-97, 1982.

[104] F.G.S. Spurrell, On the discovery of the place where Palaeolithic implements where made at Crayford. Quarterly Journal of the Geological Society of London, 36, 544-549, 1880.

[105] W.G. Smith, « Man, the primeral savage », Eduard Stanford, Londres, 319 p., 1894

[106] A. Salomon, Découverte d'un troisième gisement moustérien sur le territoire d'Hermies au lieu-dit la vallée de Bertincourt. Bulletin de la Société Préhistorique Française, 10, 191-196, 1913.

[107] V. Commont, Les terrains quaternaires des tranchées du nouveau Canal du Nord. L'Anthropologie, 27, 09-350 et 517-538, 1916 .

[108] H. Kelley, Contribution à l'étude de la technique de la taille levalloisienne. Bulletin de la Société Préhistorique Française, 51, 149-169, 1954.

[109] M. Louis, Contribution à l'étude de la taille du silex. Bulletin de la Société Préhistorique Française, 32 (12), 616-621, 1935.

[110] E. Cziesla, S. Eickhoff, N. Arts, D. Winter (eds.), « The Big Puzzle. International Symposium on Refitting Stone Artefacts », Holos, Bonn, 1990.

[111] F. Bordes, Question de contemporanéité. L’illusion des remontages. Bulletin de la Société Préhistorique française $77(5), 132-133,1980$.

[112] F. Bordes, Savez-vous remonter les cailloux à la mode de chez nous? Bulletin de la Société Préhistorique française 77 (8), 232-234, 1980.

[113] D. Cahen, Das zusammensetzen geschlagener Steinartefakte. Archäologisches Korrespondenzblatt, 6 , 81-93, 1976.

[114] D. Cahen, Question de contemporanéité : l'apport des remontages. Bulletin de la Société Préhistorique Française, 77 (8), 230-232, 1980.

[115] D. Cahen, Pour clore le débat. Bulletin de la Société Préhistorique Française, 77 (8), 234, 1980.

[116] D. Cahen, J. Moeyersons, Subsurface movements of stone artifacts and their implications for the prehistory of Central Africa. Nature, 266, 812-815, 1977. 
[117] P. Villa, Sols et niveaux d'habitat du Paléolithique inférieur en Europe et au Proche-Orient. Quaternaria, 19, 107-134, 1977.

[118] Y. Le Grand, Processus de formation des dépôts archéologiques du Pléistocène moyen de Lunel-Viel 1 (Hérault). Préhistoire Anthropologie Méditerranéennes, 3, 57-63, 1994.

[119] J.G. Bordes, La séquence aurignacienne de Caminade revisitée : l'apport des raccords d'intérêt stratigraphique. Paleo, 12, 387-407, 2000.

[120] J.B. Mallye, Badger (Meles meles) remains within caves as an analytical tool to test the integrity of stratified sites : the contribution of Unikoté cave (Pyrénées-Atlantiques, France). Journal of Taphonomy 9 (1), 15-22, 2011.

[121] A.H. Ivester, G.A. Brook, L.H. Robbins, A.C. Campbell, M.L. Murphy, E. Marais, « A sedimentary record of environmental change at Tsodilo Hills White Paintings Rock Shelter, Northwest Kalahari Desert, Botswana », dans Palaeoecology of Africa : An International Yearbook of Landscape Evolution and Palaeoenvironments, J. Runge (ed.), Taylor et Francis, Boca Raton, 53-78, 2010.

[122] S. Staurset, S. Coulson, Sub-surface movement of stone artefacts at White Paintings Shelter, Tsodilo Hills, Botswana: Implications for the Middle Stone Age chronology of central southern Africa. Journal of Human Evolution, 75, 153-165, 2014.

[123] P. Mellars, A new radiocarbon revolution and the dispersal of modern humans in Eurasia. Nature, 439 (23), 931-935, 2006.

[124] T.F.G. Higham, R.M. Jacobi, C. Bronk Ramsey, AMS radiocarbon dating of ancient bone using ultrafiltration. Radiocarbon, 48, 179-195, 2006.

[125] M. Hernandez, N. Mercier, P. Bertran, D. Colonge, L.A. Lelouvier, Premiers éléments de datation des industries du Pléistocène moyen (Acheuléen - Paléolithique moyen ancien) de la région pyrénéo-garonnaise : une approche géochronologique pluri-méthodes (TL, OSL, TT-OSL) des sites de Duclos et Romentères. Paléo, 23, 155-170, 2012.

[126] L. Bueno, J. Feathers, P. De Blasis, The formation process of a paleoindian open-air site in Central Brazil : integrating lithic analysis, radiocarbon and luminescence dating. Journal of Archaeological Science, 40, 190-203, 2013.

[127] P.C. Fanning, S.J. Holdaway, E.J. Rhodes, T.G. Bryant, The surface archaeological record in arid Australia : Geomorphic controls on preservation, exposure, and visibility. Geoarchaeology : An International Journal, 24, 121-146, 2009.

[128] T.A. Surovell, N.M. Waguenspack, J.H. Mayer, M. Kornfeld, G.C. Frison, Shallow site archaeology : artifact dispersal, stratigraphy, and radiocarbon dating at the Barger Gulch Locality B Folsom site, Middle Park, Colorado. Geoarchaeology : An International Journal, 20, 627-649, 2005.

[129] D. Stapert, Some natural surface modification on flint in the Netherlands. Palaeohistoria, 18, 7-41, 1976 .

[130] C.D. Howard, Amorphous silica, soil solutions, and archaeological flint gloss. North American Archaeologist, 20, 209-215, 1999.

[131] C.D. Howard, The gloss patination of flint artifacts. Plains anthropologist, 47, 283-287, 2002.

[132] D.B. Burroni, R.E. Donahue, A.R. Pollard, M. Mussi, The surface alteration features of flint artefacts as a record of environmental processes. Journal of Archaeological Science, 29, 1277-1287, 2002.

[133] P. Glauberman, M. Thorson, Flint patina as an aspect of "Flaked stone taphonomy" : A case study from the loess terrain of the Netherlands and Belgium. Journal of Taphonomy, 10, 1, 21-43, 2012.

[134] R.E Donahue, «The current state of lithic microwear research », dans Stories in Stone, N. Ashton, A. David (eds.), Lithic Studies Society, Oxford, London, 156-168, 1994.

[135] R.E Donahue, « Lithic microwear analysis of artefacts from Barnham », dans Excavations at the Lower Palaeolithic Site at East Farm, Barnham, Suffolk, 1989-1994, N. Ashton, S.G. Lewis, S. Parfitt (eds.), British Museum Occasional Paper, No. 125, British Museum Press, London, 245-250, 1998.

[136] D. Camuffo, Physical weathering of stones. Science of the Total Environment, 167, 1-14, 1995.

[137] J. Lancaster, Wind action on stone artifacts : An experiment in site modification. Journal of Field Archaeology, 13, 359-363, 1996.

[138] K. Borrazzo, Tafonomía lítica en dunas : una propuesta para el análisis de los artefactos líticos. Intersecciones en Antropología, 7, 247-261, 2006. 
[139] P.C. Ugalde, C.M. Santoro, E.M. Gayo, C. Latorre, S. Maldonado, R. De Pol-Holz, D. Jackson, How do surficial lithic assemblages weather in arid environments? A case study from the Atacama Desert, Northern Chile. Geoarchaeology : An International Journal, 30 (4), 352-368, 2015.

[140] K. Knutsson, K. Lindé, « Post-depositional alterations of wear marks on quartz tools. Preliminary observations of an experiment with aeolian abrasion », dans Le silex de sa genèse à l'outil. M.R. SéronieVivien, M. Lenoir (dir.), Actes du Vème colloque international sur le silex. Cahiers du Quaternaire, 17, 607-618, 1990.

[141] R. Dorn, « Rock varnish », dans Geochemical sediments and landscapes, D. Nash, S. Mclaren (eds.), Blackwell, London, 246-297, 2007.

[142] J.P. Lautridou, J.C. Ozouf, Experimental frost shattering : 15 years of research at the Centre de Géomorphologie du CNRS. Progress in Physical Geography, 6, 2, 215-232, 1982.

[143] J.P. Lautridou, Le cycle périglaciaire pléistocène en Europe du Nord-Ouest et plus particulièrement en Normandie. Thèse de Doctorat d'état, Université de Caen, 907 p., 1985.

[144] J.P. Lautridou, G. Letavernier, J.C. Ozouf, Porosité et gélivité des silex et des craies : l'apport de l'expérimentation, Actes du Xe colloque interrégional sur le Néolithique, Caen, 1983, Revue archéologique de l'Ouest, suppl. $\mathrm{n}^{\circ} 1,271-277,1986$.

[145] J.P. Lautridou, Y. Delehaye, B. Etlicher, « Expériences de gélifraction de silex », dans Le silex de sa genèse à l'outil, M.R. Séronie-Vivien, M. Lenoir (dir.), Actes du Ve colloque international sur le silex, tome 1, 1987, Bordeaux, CNRS, Cahiers du Quaternaire, 17, 161-166, 1990.

[146] L. Vallin, J.P. Caspar, G. Guillemet, B. Masson, J.C. Ozouf, 2013 : Altérations des artefacts préhistoriques en silex par les processus périglaciaires : Présentation des expériences conduites au centre de géomorphologie du CNRS de Caen. Quaternaire, 24, 3, 259-266, 2013.

[147] J.L. Guadelli, J.C. Ozouf, Etudes expérimentales de l'action du gel sur les restes fauniques : premiers résultats. Artefacts, 9, 47-56, 1994.

[148] J.L. Guadelli, La gélifraction des restes fauniques. Expérimentation et transfert au fossile. Annales de Paléontologie, 94, 121-165, 2008.

[149] J.L. Guadelli, « La gélifraction des restes fauniques. Du laboratoire au terrain », dans Messages d'os : Archéométrie du squelette animal et humain, M. Balasse, J.P. Brugal, Y. Dauphin, E.M. Geigl, Ch. Oberlin, I. Reiche (dir.). Éditions des archives contemporaines, collection Sciences Archéologiques, 177-186, 2015.

[150] J. Kaminska, E. Mycielska-Dowgialloo, K. Szymcak, « Post-depositional changes on surfaces of flint artefacts as observed under a scanning electron microscope », dans Traces et fonction : les gestes retrouvés. Anderson P., Beyries S., Otte M. et Plisson H. (dir.), Actes du Colloque international de Liège 8-10 décembre 1990, Liège, Université de Liège, ERAUL 50, 467-475, 1993.

[151] J.P. Caspar, B. Masson, L. Vallin, Poli de bois ou poli de glace au Paléolithique inférieur et moyen? Problèmes de convergence taphonomique et fonctionnelle. Bulletin de la Société Préhistorique Française, 100, 3, 453-462, 2003.

[152] E. Claud, P. Bertran, Effet de la solifluxion sur les traces d'utilisation des outils lithiques : mise en place d'une expérimentation in vivo à Gavarnie (Hautes-Pyrénées, France). Actes du XVe congrès IUSPP, Lisbonne 2006. Paléo - Supplément n³ 3, 31-42, 2010.

[153] R.C.A. Rottländer, The formation of patina on flint. Archaeometry, 17, 1, 106-110, 1975.

[154] I. Levi Sala, «Use-wear traces : processes of development and post-depositional alterations », dans Traces et fonction : les gestes retrouvés. Anderson P., Beyries S., Otte M. et Plisson H. (dir.), Actes du Colloque international de Liège 8-10 décembre 1990, Liège, Université de Liège, ERAUL 50, 401-416, 1993.

[155] H. Plisson, M. Mauger, Chemical and mechanical alteration of microwear polishes : an experimental approach. Helinium, XXVIII/ 1, 3-16, 1988.

[156] L. Vallin, B. Masson, J.P. Caspar, Taphonomy at Hermies, France : a mousterian knapping site in a loessic context. Journal of Field Archaeology, 28, 419-436, 2001.

[157] M., Shackley, 1974 : Stream abrasion of flint implements. Nature, 248, 501-502, 1974.

[158] P. Harding, P.L. Gibbard, J. Lewin, M.G. Macklin, E.H. Moss, « The transport and abrasion of flint handaxes in a gravel-bed river », dans G. Sieveking, M.H. Newcomer (eds.), The Human Uses of Flint and Chert : Proceedings of the Fourth International Flint Symposium Held at Brighton Polytechnic, Oct 15th April 1983, 115-126. Cambridge University Press, Cambridge, 1987.

[159] J.C. Chambers, Like a rolling stone? The identification of fluvial transport damage signatures on secondary context bifaces. Lithics, 24, 66-77, 2003. 
[160] R.T. Hosfield, J.C. Chambers, Flake modifications during fluvial transportation : three cautionary tales. Lithics, 24, 57-65, 2005.

[161] L. Grosman, G. Sharon, T. Goldman-Neuman, O. Smikt, U. Smilansky, Studying post depositional damage on Acheulian bifaces using 3-D scanning. Journal of Human Evolution, 60, 398-406, 2011.

[162] J.D. Clark, Acheulian occupation sites in the Middle East and Africa : A Study in cultural variability. American Anthropologist, 68, 202-229, 1966.

[163] J.J. Wymer, Lower Palaeolithic archaeology in Britain as represented by the Thames Valley. John Baker, London, 429 p., 1968.

[164] J.J. Wymer, « The Interpretation of Palaeolithic cultural and faunal material found in Pleistocene sediments », dans Geoarchaeology : earth science and the past, D.A. Davidson, M.L. Shackley (eds.), Duckworth, London, 327-334, 1976.

[165] R. Singer, J.J. Wymer, B.G. Gladfelter, R.G. Wolff, Excavation of the Clactonian industry at the golf course, Clacton-on-Sea, Essex. Proceedings of the Prehistoric Society, 39, 6-74, 1973.

[166] J.D. Clark, H. Kurashina, « New plio-Pleistocene archaeological occurrences from the plain of Gadeb, upper Webi Shebele basin, Ethiopia, and a statistical comparison of the Gadeb sites with other early stone age assemblages », dans The Earlier Industries of Africa, J.D. Clark, G. Isaac (eds.), IUPPS congress 9, colloque 5, Nice, 158-216, 1976.

[167] M.D. Petraglia, R. Potts, Water flow and the formation of early Pleistocene artifact sites in Olduvai Gorge, Tanzania. Journal of Anthropological Archaeology, 13, 228-254, 1994.

[168] R.T. Hosfield, « Rolling stones : understanding river-rolled Paleolithic artifact assemblages », dans Geoarchaeology, Climate Change, and Sustainability, A.G. Brown, L.S. Basell, K.W. Butzer (eds.), Geological Society of America Special Paper, 476, Boulder, 37-52, 2011.

[169] W. Chu, No stone left unturned : fluvial processes in the Pleistocene of northern Europe. Ph.D Thesis, University of Reading, 428 p., 2013.

[170] W. Chu, Thompson C., Hosfield R.T., Micro-abrasion of flint artifacts by mobile sediments : a taphonomic approach. Archaeological and Anthropological Sciences, DOI 10.1007/s12520-013-0157-0, 2013.

[171] R.T. Hosfield, J.C. Chambers, M.G. Macklin, P. Brewer, D. Sear, Interpreting secondary context sites : a role for experimental archaeology. Lithics, 21, 29-35, 2000.

[172] R.T. Hosfield, J.C. Chambers, Flake modifications during fluvial transportation : three cautionary tales. Lithics, 24, p. 57-65, 2003.

[173] R.T. Hosfield, J.C. Chambers, Experimental archaeology on the Afon Ystwyth, Wales, UK. Antiquity, 78, 299, http ://antiquity.ac.uk/ProjGall/chambers/index.html., 2004.

[174] J.C. Chambers, «River gravels and handaxes : New experiments in site formation, stone tool transportation and transformation », dans Experimentelle Archäologie in Europa, M. Fansa (ed.), Bilanz 2004, Heft 3, Isensee Verlag, Oldenburg, 25-41, 2005.

[175] A.G. Brown, Alluvial geoarchaeology : Floodplain archaeology and environmental change. Cambridge University Press, Cambridge, 377 p., 1997.

[176] M. Shackley, A study of the Mousterian of Acheulian tradition industries of Southern Britain. Unpublished Ph.D. Thesis, University of Southampton, 1975.

[177] R.T. Hosfield, J.C. Chambers, « River gravels and flakes : new experiments in site formation, stone tool transportation and transformation », dans Experimentelle Archäologie in Europa, M. Fansa (ed.), Bilanz 2004, Heft 3, Isensee Verlag, Oldenburg, 57-74, 2005.

[178] H. Bunn, J.W.K. Harris, G. Isaac, Z. Kaufulu, E. Kroll, K. Schick, N. Toth, A.K. Behrensmeyer, FxJj50 : an Early Pleistocene site in northern Kenya. World Archaeology, 12, 109-136, 1980.

[179] P.J. Texier, Désilicification des silex taillés. Quaternaria, 23, 159-169, 1981.

[180] S. Pappu, A study of natural site formation processes in the Kortallayar Basin, Tamil Nadu, South India. Geoarchaeology : An International Journal, 14, 127-150, 1999.

[181] R.C.A. Rottländer, Verwitterungserscheinungen an Keramik, Silices und Knochen, Teil 2 : Verwitterungserscheinungen an Silices und Knochen. Tübinger Beiträge zur Archäometrie 3. Verlag Archaeologica Venatoria, Institute für Urgeschichte der Universität Tübingen, Tübingen, 1989. 
[182] H. Plisson, « Altération des micropolis d'usage : quelques expériences complémentaires », dans Technical Aspects of Microwear Studies on Stone Tools, L.R. Owen, G. Unrath (dir.), Actes de la conférence de Tübingen, 1985, Early Man News, 9-10-11, 111-116, 1986.

[183] B.A. Purdy, D.E. Clark, Weathering of inorganic materials : dating and other applications. Advances in Archaeological Method and Theory, 11, 211-253, 1987.

[184] P. Hinsinger, O.N. Fernandes Barros, M.F. Benedetti, Y. Noack, G. Callot, Plant-induced weathering of a basaltic rock : experimental evidence. Geochimica et Cosmochimica Acta, 65, 137-152, 2001.

[185] M. Thiry, P. Fernandes, A. Milnes, J.P. Raynal, Driving forces for the weathering and alteration of silica in the regolith : Implications for studies of prehistoric flint tools. Earth-Science Reviews, 141-154, 2014.

[186] M.A. Summerfield, Global geomorphology. Wiley and Sons, Longman Scientific and Technical, 537 p., 1991.

[187] M.R. Aubry, Y. Dewolf, T. Muxart, Altérations des silex de la craie : étude expérimentale, premières observations. Comptes Rendus de l'Académie des Sciences de Paris, 280, D, 1509-1512, 1975.

[188] L.H. Keeley, Experimental determination of stone tool uses : a microwear analysis. The University of Chicago Press, Prehistoric Archaeology and Ecology, Chicago, 226 p., 1980.

[189] H. Plisson, De la conservation des micropolis d'utilisation. Bulletin de la Société préhistorique française, 80, 74-77, 1983.

[190] R. Baesemann, « Natural alterations of stone artefact materials », dans Technical aspects of microwear studies on stone tools, Owen L.R., Unrath G. (dir.), Actes de la conférence de Tübingen, février 1985. Early Man News 9-10-11, 97-102, 1986.

[191] A. Goodwin, « Chemical alteration (patination) of stone », dans The Application of Quantitative Methods in Archaeology, R. Heizer, S. Cook (eds.). Quadrangle Books, Chicago, 300-324, 1960.

[192] V.J. Hurst, A.R. Kelly, Patination of Cultural Flints. Science, 134/3474, 251-256, 1961.

[193] J. Van Nest, Patination of knife river flint artifacts. Plains Anthropologist, 30, 325- 339, 1985.

[194] P. Sheppard, L. Pavlish, Weathering of archaeological cherts : A Case Study from the Solomon Islands. Geoarchaeology : An International Journal, 7, 41-53, 1992. 